\title{
PRODUCT MEASURES
}

\author{
BY \\ W. W. BLEDSOE AND A. P. MORSE
}

1. Introduction. One of our aims is to free topological Fubini theory from the usual restraints of local compactness. In this connection we shall regard Radon measures as very special indeed. Another of our aims is to associate with any two measures $\mu$ and $\nu$ such a product measure $\phi$ that in the event $\mu$ and $\nu$ happen to be topological measures of a rather general sort, then our topology-free measure $\phi$ will actually be a similar topological measure under which the Borel subsets of the product space are measurable( $\left.{ }^{1}\right)$. We shall reach both these goals even though an obvious obstacle in our path to the second is the fact that the open sets of a topological product are not always contained in the $\sigma$-field generated by the open rectangles. The product measure we construct in order to overcome this difficulty allows more summable functions and greater freedom of action than the product measures considered heretofore.

In $\$ 3$ we consider a family of sets which is closed to finite union and countable nonvacuous intersection. With this family we associate a family of measures and for these measures we infer subset theorems which yield at once, in $\$ 6$, corresponding closed subset theorems.

$\$ 4$ deals with some aspects of measure integration and gives a fundamental result, 4.10 , on the approximation of integrable functions by functions of a more elementary character.

In $\S 5$ we develop a general Fubini theory and construct a specific product measure.

In $\$ 6$ topology enters our theory for the first time. Several kinds of topological measures are considered; applications of $\$ 3$ are made; the reactions of various measures to a sort of taming process are investigated in 6.8 ; locally null sets are examined briefly; and finally some attention is paid to metric measures.

$\S 7$, with which we close our paper, is devoted to a topological Fubini theory in which the nontopological product measure constructed in $\$ 5$ not only has considerable topological merit in its own right, but also prototypes two other interesting, topologically fashioned, product measures.

To many our result of greatest interest and utility will be Theorem 7.7 viewed in the light of 5.11.3. Because of the technical language in which 7.7 is phrased, we should like to give here a somewhat detailed but informal account of much of its content.

Presented to the Society, August 31, 1954; received by the editors May 15, 1954.

(1) Whether or not ordinary product measure has this property remains an open question. 
Suppose $\mu$ is an (outer) measure defined for all the subsets of a topological space $\delta$. Suppose that $\mu(\delta)<\infty$ and that each open set is not only measurable but equal in measure to the upper bound of the measures of closed subsets. Suppose further that from each covering of $S$ by open sets a countable subfamily can be extracted which covers almost all of $S$. If $\nu$ is another measure like $\mu$, then our associated product measure bears to the topological product space the same relation as $\mu$ does to $\delta$, and at the same time satisfies the Fubini equality for summable functions. The novel feature of our product measure is that we require to be of measure zero each set whose characteristic function integrates iteratively in both orders to zero.

Any reader whose interest in the present paper does not extend beyond 5.11 and 7.7 is advised to omit all of $\$ 3$ except 3.1-3.6 and all of $\S 6$ except $6.0,6.2 .1,6.2 .5,6.4,6.9-6.12$. Among the results so bypassed is Theorem 6.3.2 whose applicability to a class of sets far more extensive than analytic sets can scarcely escape anyone interested in the latter.

\section{Preliminary definitions and notations.}

2.1 Definitions.

$.1 \sim B=$ the complement of $B$.

.2 sng $y=\mathrm{E} x(x=y)$.

Thus sng $y$ is the set whose sole member is $y$.

$.3 \sigma \mathfrak{F}=\mathrm{U} \beta \in \mathfrak{F} \beta=\mathrm{Ex}(x \in \beta$ for some $\beta \in \mathfrak{F})$.

$.4 \pi \mathfrak{F}=\cap \beta \in \mathfrak{F} \beta=\mathrm{E} x(x \in \beta$ for each $\beta \in \mathfrak{F})$.

$.5 \mathrm{sb} A=$ subset $A=\mathrm{E} \beta(\beta \subset A)$.

$.6 \operatorname{sp} A=$ superset $A=\mathrm{E} \beta(\beta \supset A)$.

2.2 Definitions.

$.1 \mathrm{dmn} f=\operatorname{domain} f=\operatorname{Ex}[(x, y) \in f$ for some $y]$.

$.2 \mathrm{dmn}^{\prime} f=\mathrm{E} t \in \operatorname{dmn} f[|f(t)|<\infty]$.

$.3 \mathrm{rng} f=\operatorname{range} f=\operatorname{E} y[(x, y) \in f$ for some $x]$.

$.4 \operatorname{rim} \phi=\operatorname{realm} \phi=\sigma \mathrm{dmn} \phi$.

In the above $f$ and $\phi$ are not required to be functions; they may equally well be relations (i.e., sets of ordered pairs) of which functions are but special cases. We assume, of course, there is no difference between a function and its graph.

\subsection{Definitions.}

$.1 \mathrm{rct} A B=$ the set of ordered pairs $(x, y)$ such that $x \in A$ and $y \in B$.

.2 Rct $\mathfrak{F}(S)=\mathrm{E} R(R=\operatorname{rct} A B$ for some $A \in \mathfrak{F}$ and some $B \in(\mathfrak{F})$.

2.4 Definitions.

$.1 \omega^{\prime}=$ the set of integers.

$.2 \omega=$ the set of non-negative integers.

We shall assume the integer 0 and the empty set are the same.

2.5 Definition. $\mathrm{cmpl} \mathfrak{F}=\mathrm{E} B(B=\sigma \mathfrak{F} \sim C$ for some $C \in \mathfrak{F})$.

3. Measures.

3.1 Definitions. 
$.1 \phi$ measures $\delta$ if and only if $\phi$ is such a function on $\mathrm{sb} S$ that:

$$
0 \leqq \phi(A) \text { whenever } A \subset \mathcal{S} \text {; }
$$

and

$$
\phi(A) \leqq \sum B \in \mathfrak{F} \phi(B)
$$

whenever $\mathfrak{F}$ is a countable family for which

$$
A \subset \sigma \mathfrak{F} \subset \mathrm{S} \text {. }
$$

Clearly if $\phi$ measures both $S$ and $S^{\prime}$ then $S=S^{\prime}$.

$.2 \mathrm{Msr} \delta=\mathrm{E} \phi(\phi$ measures $\delta)$.

We now use 2.2.4.

.3 Measure $=\mathrm{E} \phi(\phi$ measures $\operatorname{rlm} \phi)$.

$.4 \mathrm{mbl} \phi=$ measurable $\phi=\mathrm{E} A \in \mathrm{dmn} \phi[\phi \in$ Measure and

$$
\phi(T)=\phi(T A)+\phi(T \sim A)
$$

whenever $T \in \mathrm{dmn} \phi]$.

We now use 2.2.2.

$.5 \mathrm{mbl}^{\prime} \phi=\mathrm{mbl} \phi \cap \mathrm{dmn}^{\prime} \phi$.

.6 sct $\phi T=$ section $\phi T=$ the function $\psi$ on $\mathrm{dmn} \phi$ such that, for each $A \in \mathrm{dmn} \phi$,

$$
\psi(A)=\phi(T A) .
$$

.7 sms $\phi=$ submeasure $\phi=\mathrm{E} \psi[\phi \in$ Measure and $\psi=$ sct $\phi T$ for some $\left.T \in \mathrm{dmn}^{\prime} \phi\right]$.

$.8 \mathrm{mss} g \mathcal{S} \mathfrak{S}=$ the function $\phi$ on sb $\mathcal{S}$ such that if $A$ is any subset of $\mathcal{S}$ then $\phi(A)$ is the infimum of numbers of the form

$$
\sum B \in \mathfrak{F} g(B)
$$

where $\mathfrak{F}$ is such a countable subfamily of $\mathfrak{W}$ that $A \subset \sigma \mathfrak{F}$.

In this connection we should like to remind the reader that an empty infimum is $\infty$.

Many special instances of the construction described in .8 have been given. Not unexpected, therefore, and certainly not unknown is the following general theorem.

3.2 THEOREM. If $\mathcal{S}$ is a space, $\mathfrak{E}$ is a family of sets, $g$ is a function on $\mathfrak{E}$ to the non-negative reals, then

$$
\operatorname{mss} g \mathcal{S} \mathfrak{S} \in \operatorname{Msr} \mathcal{S} .
$$

Those measure theoretic results of which we presuppose a knowlege may be found in H. Hahn, Theorie der reellen Functionen, vol. 1, Berlin, 1921, pp. 424-432. 


\subsection{Definitions.}

.1 borel $\mathfrak{F}=$ the smallest family which contains $\mathfrak{F} \cup \operatorname{sng} \sigma \mathfrak{F}$ and which is closed to countable (including vacuous) union and nonvacuous countable intersection.

.2 Borel $\mathfrak{F}=$ the smallest family which contains $\mathfrak{F}$ and which is closed to countable (including vacuous) union and complementation with respect to $\sigma \mathfrak{F}$.

3.4 Definition. internal $=\mathrm{E} \mathfrak{F}[\mathfrak{F}$ is closed to finite (including vacuous) union and nonvacuous countable intersection].

3.5 Definition. pron $\phi \mathfrak{F}=\mathrm{E} B[\phi \in$ Measure and

$$
\inf C \in \mathfrak{F} \cap \mathrm{sb} B \phi(B \sim C)=0] .
$$

Thus prxn $\phi \mathfrak{F}$ consists of those sets which can be $\phi$-approximated from the inside by members of $\mathfrak{F}$.

In 3.6.3 below we introduce a concept which has received relatively little attention. To guide our intuition we glance ahead at 6.0 and then imagine $\mathfrak{T} \in$ topology and $\mathfrak{F}=$ Closed $\mathfrak{T}$.

3.6 Definitions.

.1 Hull $\mathfrak{G}=\mathrm{E} \phi \in \mathrm{Msr} \sigma \mathfrak{S}\left[\right.$ Each $A \in \mathrm{dmn} \phi$ is so contained in some $A^{\prime} \in \mathfrak{W}$ that $\left.\phi(A)=\phi\left(A^{\prime}\right)\right]$.

.2 Mass $\mathfrak{F}=\mathrm{E} \phi \in \mathrm{Msr} \sigma \mathfrak{F}[\mathfrak{F} \in$ internal $\cap \mathrm{sb} \operatorname{mbl} \phi$, and $\mathrm{cmpl} \mathfrak{F} \subset \operatorname{prxn} \psi \mathfrak{F}$ whenever $\psi \in \operatorname{sms} \phi]$.

.3 Bore $\mathfrak{F}=\mathrm{E} A \subset \sigma \mathfrak{F}[A \in \mathrm{mbl} \phi$ whenever $\phi \in$ Mass $\mathfrak{F}]$.

3.7 TheOREMS.

.1 borel $\mathfrak{F} \subset$ Borel $\mathfrak{F} \subset$ Bore $\mathfrak{F}$.

.2 If $\phi \in$ Mass $\mathfrak{F}$ then Bore $\mathfrak{F} \subset \mathrm{mbl} \phi$.

.3 If $\phi \in$ Mass $\mathfrak{F}$ then sms $\phi \subset$ Mass $\mathfrak{F}$.

.4 If $\phi \in \mathrm{Msr} \sigma \mathfrak{F}, \mathfrak{F} \in$ internal $\cap \mathrm{sb} \mathrm{mbl} \phi$, and $\mathrm{cmpl} \mathfrak{F} \subset \operatorname{prxn} \phi \mathfrak{F}$, then $\phi \in$ Mass $\mathfrak{F}$.

.5 Mass Borel $\mathfrak{F}=\mathrm{E} \phi \in \mathrm{Msr} \sigma \mathfrak{F}(\mathfrak{F} \subset \mathrm{mbl} \phi)$.

.6 Bore Borel $\mathfrak{F} \subset$ Bore $\mathfrak{F}$.

A well-known, useful, and fairly obvious companion to 3.2 is

3.8 Theorem. If $\mathcal{S}=\sigma \mathfrak{S}, \phi \in \mathrm{Msr} \mathcal{S}$,

$$
\mathfrak{S}=\text { Borel } \mathfrak{E} \subset \mathrm{mbl} \phi,
$$

and

$$
\psi=\operatorname{mss} \phi S \mathfrak{S},
$$

then:

$.1 \psi \in \mathrm{Msr} S$;

.2 if $A \subset S$ then $\phi(A) \leqq \psi(A)$;

.3 if $A \in \mathfrak{S}$ then $\phi(A)=\psi(A)$; 
$.4 \psi \in$ Hull $\mathfrak{S}$;

$.5 \mathfrak{g} \subset \mathrm{mbl} \psi$.

Also of use to us is

3.9 Theorem. If $\phi \in$ Hull $\mathfrak{S}, \mathfrak{S}=$ Borel $\mathfrak{E}$, and

$\mathfrak{S} \cap \mathrm{dmn}^{\prime} \phi \subset \operatorname{prxn} \phi \mathfrak{F}$,

then

$$
\operatorname{mbl}^{\prime} \phi \subset \operatorname{prxn} \phi \mathfrak{F} .
$$

Proof. The desired conclusion is evidently a consequence of the

Statement. If $r>0$ and $B \in \mathrm{mbl}^{\prime} \phi$ then there is $a C \in \mathfrak{F}$ for which

$$
C \subset B \text { and } \phi(B \sim C) \leqq r .
$$

Proof. So choose $B^{\prime}$ and $B^{\prime \prime}$ from $\mathfrak{S}$ that

$$
\begin{array}{cl}
B \subset B^{\prime}, & B^{\prime} \sim B \subset B^{\prime \prime}, \\
\phi\left(B^{\prime}\right)=\phi(B), & \phi\left(B^{\prime \prime}\right)=\phi\left(B^{\prime} \sim B\right) .
\end{array}
$$

Notice that

$$
B \sim B^{\prime \prime}=B^{\prime} \sim B^{\prime \prime} \in \mathfrak{G} \cap \mathrm{dmn}^{\prime} \phi
$$

and then use the fact that

$$
\mathfrak{S} \cap \mathrm{dmn}^{\prime} \phi \subset \operatorname{prxn} \phi \mathfrak{F}
$$

to so secure $C \in \mathfrak{F}$ that

$$
C \subset B \sim B^{\prime \prime} \text { and } \phi\left(\left(B \sim B^{\prime \prime}\right) \sim C\right) \leqq r .
$$

Thus we see that $C \subset B$, and, since $B \in \mathrm{mbl}^{\prime} \phi$, we conclude

$$
\begin{aligned}
\phi(B \sim C) & \leqq \phi\left(\left(B \sim B^{\prime \prime}\right) \sim C\right)+\phi\left(B^{\prime \prime}\right) \\
& \leqq r+\phi\left(B^{\prime \prime}\right) \\
& =r+\phi\left(B^{\prime} \sim B\right) \\
& =r+\phi\left(B^{\prime}\right)-\phi(B) \\
& =r+0=r .
\end{aligned}
$$

Lemma 3.10.1 below is not hard to check and is presumably known to many. Lemma 3.10.2 was known to W. Sierpinski, Les ensembles Boreliens abstraits, Annales de la Société Polanaise de Mathématique vol. 6 (1927) p. 51. It can be readily verified either directly or with the help of A. P. Morse, The role of internal families in measure theory, Bull. Amer. Math. Soc. vol. 50 (1944) p. 724, Theorem 3.2. Lemma 3.11 is a consequence of Part I of the proof of 4.5 on p. 725 of the paper just cited. 
3.10 Lemmas.

.1 If $\mathrm{cmpl} \mathfrak{F} \subset$ borel $\mathfrak{F}$ then $\mathfrak{F}$ contains such a countable subfamily $\mathfrak{F}^{\prime}$ that $\sigma \mathfrak{F}^{\prime}=\sigma \mathfrak{F}$.

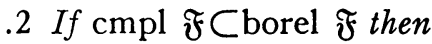

$$
\text { borel } \mathfrak{F}=\text { Borel } \mathfrak{F} \text {. }
$$

3.11 Lemma. If $\phi \in \mathrm{Msr} \sigma \mathfrak{F}, \phi(\sigma \mathfrak{F})<\infty$, $\mathfrak{F} \in$ internal $\cap \mathrm{sb} \mathrm{mbl} \phi$, and $\mathfrak{S}$ is a countable nonvacuous subfamily of prxn $\phi \mathfrak{F}$ then $\sigma \mathfrak{S} \in \operatorname{prxn} \phi \mathfrak{F}$ and $\pi \mathfrak{S}$ $\in \operatorname{prxn} \phi \mathfrak{F}$.

3.12 Theorem. If $\phi \in \mathrm{Msr} \sigma \mathfrak{F}, \phi(\sigma \mathfrak{F})<\infty, \mathfrak{F} \in$ internal $\cap \mathrm{sb} \mathrm{mbl} \phi$,

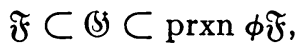

then

$$
\text { borel } \leftrightarrow \subset \text { prxn } \phi \mathfrak{F} \cup \text { sng } \sigma \mathfrak{F} \text {. }
$$

Proof. Note that $\sigma \mathfrak{F}=\sigma \mathfrak{S}, 0 \in \mathfrak{F}$, and then apply 3.11 .

By taking $\mathfrak{F}=\$ S$ in 3.12 and then using 3.10 .1 and 3.11 we easily infer

3.13 Theorem. If $\mathfrak{F} \in$ internal and $\mathrm{cmpl} \mathfrak{F} \subset$ borel $\mathfrak{F}$ then

$$
\text { Mass } \mathfrak{F}=\mathrm{E} \phi \in \operatorname{Msr} \sigma \mathfrak{F}[\mathfrak{F} \subset \mathrm{mbl} \phi] .
$$

3.14 Lemma. If $\phi \in$ Mass $\mathfrak{F}$ and $\phi(\sigma \mathfrak{F})<\infty$ then

$$
\text { Borel } \mathfrak{F} \subset \text { prxn } \phi \mathfrak{F} \text {. }
$$

Proof. Let

$$
B=\mathfrak{F} \cup \operatorname{compl} \mathfrak{F}
$$

Since

$$
0 \in \mathfrak{F}, \quad \operatorname{cmpl} \mathfrak{F} \subset \operatorname{prxn} \phi \mathfrak{F}
$$

it is clear that

$$
\sigma \mathfrak{F} \in \operatorname{prxn} \phi \mathfrak{F}, \quad \mathfrak{F} \subset \mathcal{B} \subset \operatorname{prxn} \phi \mathfrak{F} .
$$

From this and the fact that $B=\mathrm{cmpl}(S)$ it now follows from 3.10 .2 and 3.12 that

$$
\begin{aligned}
\text { Borel } \mathfrak{F} & \subset \text { Borel } \mathfrak{H}=\text { borel } \mathfrak{S} \\
& \subset \operatorname{prxn} \phi \mathfrak{F} \cup \operatorname{sng} \sigma \mathfrak{F}=\operatorname{prxn} \phi \mathfrak{F} .
\end{aligned}
$$

Theorems 3.15 and 3.16 below were given by A. P. Morse in his 1952-53 Lectures on Real Variable at the University of California.

3.15 Theorem. If $\phi \in$ Mass $\mathfrak{F}$ then 
Bore $\mathfrak{F} \cap \mathrm{dmn}^{\prime} \phi \subset \operatorname{prxn} \phi \mathfrak{F}$.

Proof. We suppose

$$
B \in \text { Bore } \mathfrak{F} \cap \mathrm{dmn}^{\prime} \phi
$$

and complete the proof by showing

$$
B \in \operatorname{prxn} \phi \mathfrak{F} \text {. }
$$

Let

$$
\begin{aligned}
\mathfrak{S} & =\sigma \mathfrak{F}, \quad \mathfrak{W}=\text { Borel } \mathfrak{F}, \quad \phi^{\prime}=\operatorname{sct} \phi B \\
\psi & =\operatorname{mss} \phi^{\prime} \mathcal{S} \mathfrak{E},
\end{aligned}
$$

and notice

$$
\psi(\delta)=\phi^{\prime}(\delta)=\phi(B)<\infty .
$$

Because of 3.7.3 we know $\phi^{\prime} \in$ Mass $\mathfrak{F}$. Consequently 3.14 assures us $\mathfrak{S} \subset \operatorname{prxn} \phi^{\prime} \mathfrak{F}$

Clearly

$$
\mathfrak{S}=\text { Borel } \mathfrak{G} \subset \mathrm{mbl} \phi^{\prime} .
$$

With 3.8 in mind we now infer: from 3.8.3 that

$$
\text { cmpl } \mathfrak{F} \subset \mathfrak{S} \subset \operatorname{prxn} \psi \mathfrak{F} ;
$$

from 3.8.5 that

$$
\mathfrak{F} \subset \mathfrak{S} \subset \operatorname{mbl} \psi ;
$$

from 3.6.2 that

$$
\text { i., } \quad \mathfrak{F} \in \text { internal; }
$$

from (1), (2), (3), and 3.7.4 that

$$
\psi \in \text { Mass } \mathfrak{F}
$$

from (4) and 3.7.2 that

$$
B \in \mathrm{mbl} \psi=\mathrm{mbl}^{\prime} \psi ;
$$

from (5), (1), 3.8.4, and 3.9 that

$$
B \in \operatorname{mbl}^{\prime} \psi \subset \operatorname{prxn} \psi \mathfrak{F} ;
$$

from (6), 3.5, and 3.8.2 that corresponding to each $r>0$ there is a $C \in \mathfrak{F} \cap \operatorname{sb} B$ for which

$$
r \geqq \psi(B \sim C) \geqq \phi^{\prime}(B \sim C)=\phi(B \sim C) .
$$


Consequently

$$
B \in \operatorname{prxn} \phi \mathfrak{F}
$$

and the proof is complete.

3.16 Theorem. If $\phi \in$ Mass $\mathfrak{F} \cap$ Hull Bore $\mathfrak{F}$ then

$$
\operatorname{mbl}^{\prime} \phi \subset \operatorname{prxn} \phi \mathfrak{F} \text {. }
$$

Proof. Let $\mathfrak{S}=$ Bore $\mathfrak{F}$. Notice that

$$
\mathfrak{E}=\text { Borel } \mathfrak{S} \text {, }
$$

infer from 3.15 that

$$
\mathfrak{S} \cap \mathrm{dmn}^{\prime} \phi \subset \operatorname{prxn} \phi \mathfrak{F},
$$

and conclude from 3.9 that

$$
\operatorname{mbl}^{\prime} \phi \subset \operatorname{prxn} \phi \mathfrak{F} .
$$

4. Integrable functions. We have no intention of developing measure integration here. However we do wish to make clear certain fundamental aspects of this subject as we presently view it.

4.0 Definition. $\mathrm{cmbl} \phi=\mathrm{E} A\left[\mathrm{mbl}^{\prime} \phi\right.$ contains such a countable family $\mathfrak{F}$ that $A=\sigma \mathfrak{F}]$.

\subsection{Definitions.}

.1 Massable $\phi=\mathrm{E} f[\phi \in$ Measure, $f$ is such a function on some subset of $\operatorname{rlm} \phi$ to the reals that

$$
\mathrm{E} x[f(x) \geqq \lambda] \in \mathrm{mbl} \phi
$$

whenever $-\infty \leqq \lambda \leqq \infty]$.

.2 Integrable $+\phi=\mathrm{E} f \in$ Massable $\phi[f$ is such a function on $\operatorname{rlm} \phi$ to the non-negative reals that

$$
\mathrm{E} x[f(x)>0] \in \mathrm{cmbl} \phi] .
$$

$.3 \mathrm{Alm} \phi x P$ if and only if $\phi \in$ Measure and $\phi(\operatorname{rlm} \phi \sim \mathrm{Ex} P)=0$.

In .3 above we allow ' $P$ ' to be replaced by an arbitrary formula such as for example

$$
\text { ' }[f(x, y) \geqq 0] \text { '. }
$$

4.2 Remark. There are, of course, many different approaches to measure integration. The approach we have in mind, but do not specify, leads to an integral which enjoys properties .1 through .5 listed below. On a purely factual basis any approach to integration which yields properties .1 through .5 is equally satisfactory for our immediate purposes. We do not, however, recommend .1 through .5 as an approach to integration. 
Properties .2 and .3 tell us the sort of integrand to expect in an existent integral. Properties .4 and .5 help us evaluate the integral of certain integrands. Property .1 is useful on both counts.

We assume, henceforth, that $\infty-\infty$ is not a number and we also assume that

$$
0 \cdot y=y \cdot 0=0
$$

no matter what $y$ may be, but that $x$ and $y$ are always numbers whenever $x \pm y$ is a number.

.1 If $\operatorname{Alm} \phi x\{f(x)=g(x)\}$ then

$$
\int f(x) \phi d x=\int g(x) \phi d x .
$$

.2 If $\phi \in$ Measure and $\int f(x) \phi d x$ is a number, then

$$
\text { Alm } \phi x\{f(x) \text { is a number }\} \text {. }
$$

.3 If $\phi \in$ Measure and $f$ is such a function on $\operatorname{rlm} \phi$ to the non-negative reals that $\int f(x) \phi d x$ is a number, then $f \in$ Integrable $+\phi$.

Definition 2.4 .1 is used in .4 below.

.4 If $f \in$ Integrable $+\phi$, then

$$
\begin{aligned}
\int f(x) \phi d x= & \infty \cdot \phi(\mathrm{E} x[f(x)=\infty]) \\
& +\lim _{\lambda \rightarrow 1+} \sum n \in \omega^{\prime}\left\{\lambda^{n} \cdot \phi\left(\mathrm{E} x\left[\lambda^{n} \leqq f(x)<\lambda^{n+1}\right]\right)\right\} .
\end{aligned}
$$

.5 If $f \in$ Massable $\phi$, then

$$
\int f(x) \phi d x=\int P(x) \phi d x-\int N(x) \phi d x
$$

where $P$ and $N$ are such functions on $\operatorname{rlm} \phi$ that

$$
P(x)=\operatorname{Inf} \mathrm{E} t[0 \leqq t \geqq f(x)]
$$

and

$$
N(x)=\operatorname{Inf} \mathrm{E} t[0 \leqq t \geqq-f(x)]
$$

whenever $x \in \operatorname{rlm} \phi$.

In connection with .5 it should be clear that if

$$
\alpha=\operatorname{Inf}[0 \leqq t \geqq \lambda]
$$

then:

if $0 \leqq \lambda$ then $\alpha=\lambda$;

if $\lambda \leqq 0$ then $\alpha=0$; 
if $\lambda$ is not a real number then $\alpha=\infty$.

Largely because of .3 and 4.1.2 it turns out that if $\phi \in M s r S$, then

$$
\int 1 \phi d x=\phi(\delta)
$$

if and only if

$$
S \in \mathrm{cmbl} \phi .
$$

Thus in peripheral situations an integral different from ours may well be $\infty$ whereas ours is not even a number. We feel, however, that this slight lack of generality is more than made up for by a more attractive theory. For example, in terms of a slightly more general integral than ours, Theorem 5.11 could be viewed in the light of Definition 5.0 .8 and then refuted by a well known counter-example given by S. Saks at the bottom of page 87 of his Theory of the integral, New York, 1937. We avoid such difficulties by simply refusing to attempt the integration of any function which cannot itself be approximated almost everwhere by a sequence of functions with finite valued integrals.

4.3 Definitions.

.1 Integrable $\phi=\mathrm{E} f \in$ Massable $\phi\left[-\infty \leqq \int f(x) \phi d x \leqq \infty\right]$.

.2 Summable $\phi=E f \in$ Integrable $\phi\left[-\infty<\int f(x) \phi d x<\infty\right]$.

.3 Summable $+\phi=$ Integrable $+\phi \cap$ Summable $\phi$.

To avoid tedious future reasoning by cases it is convenient to have available once and for all the following theorem. then

4.4 ThEOREM. If $0 \leqq c \leqq \infty, A l m \phi x\{f(x) \geqq 0\}$, and $\int f(x) \phi d x$ is a number,

$$
0 \leqq c \cdot \int f(x) \phi d x=\int\{c \cdot f(x)\} \phi d x \leqq \infty .
$$

4.5 Definitions.

.1 cps $\mathfrak{F}=\operatorname{E} \lambda(\lambda$ is a function, $\operatorname{dmn} \lambda=\mathfrak{F}, 0 \leqq \lambda(\beta) \leqq \infty$ whenever $\beta \in \mathfrak{F}$, $\mathrm{E} \beta[\lambda(\beta)>0]$ is countable).

$.2 \operatorname{Cr} x A=1$ or 0 according as $x$ is or is not a member of $A$.

.3 Crct $S \mathfrak{F}=\mathrm{E} f(f$ is such a function on $S$ that, for some $\lambda \in \operatorname{cps} \mathfrak{F}$,

$$
f(x)=\sum \beta \in \mathfrak{F}\{\lambda(\beta) \cdot \operatorname{Cr} x \beta\}
$$

whenever $x \in S)$.

In connection with .3 we do not assume $\mathfrak{F}$ is countable. Moreover

$$
\sum \beta \in \mathfrak{F}\{\lambda(\beta) \cdot \operatorname{Cr} x \beta\}
$$

is the supremum of numbers of the form

$$
\sum \beta \in \mathfrak{F}^{\prime}\{\lambda(\beta) \cdot \operatorname{Cr} x \beta\}
$$


where $\mathfrak{F}^{\prime}$ is a finite subset of $\mathfrak{F}$.

$.4 \mathrm{bsc} \phi=\mathrm{E} \mathfrak{F} C \mathrm{cmbl} \phi(\phi \in$ Measure and $\phi=\operatorname{mss} \phi \operatorname{rlm} \phi \mathfrak{F})$.

For example, if $\phi$ is Lebesgue measure and $\mathfrak{F}$ is the family of open intervals, then $\mathfrak{F} \in$ bsc $\phi$.

Theorem 4.10 below was given by A. P. Morse in his 1946-1947 Lectures on Real Variable at the University of California. We feel it is a convenient and useful tool and since we are unaware of its appearance elsewhere we shall sketch a proof.

As was indicated above, it is quite possible that

$$
\mathfrak{F} \in \operatorname{bsc} \phi
$$

and yet for the members of $\mathfrak{F}$ to be so simple in structure that it is comparatively easy to acquire information about members of

Crct rlm $\phi \mathfrak{F}$.

This information may sometimes then be converted, by means of 4.10 , into interesting knowledge about members of Integrable $\phi$. It is in this way that we attack our general Fubini Theorem 5.3.

Fairly evident are

4.6 Theorems.

.1 If $\mathfrak{F} \in \mathrm{bsc} \phi$ and $\mathcal{S}=\operatorname{rlm} \phi$ then

$$
\text { Crct } \lesssim \mathfrak{F} \subset \text { Integrable }+\phi .
$$

.2 If $\mathfrak{F} \in \operatorname{bsc} \phi, \mathcal{S}=\operatorname{rlm} \phi, r>0, f \in$ Summable $+\phi$, then there is such $a$ member $g$ of Crct $\mathcal{S} \mathfrak{F}$ that

$$
f(x) \leqq g(x) \quad \text { whenever } x \in \mathcal{S} \text {, }
$$

and

$$
\int g(x) \phi d x \leqq \int f(x) \phi d x+r .
$$

By considering positive and negative parts we readily obtain

4.7 Lemma. If $\mathfrak{F} \in \mathrm{bsc} \phi, \mathcal{S}=\operatorname{rlm} \phi, r>0, f \in$ Summable $\phi$, then there are such members $g$ and $h$ of Crct $\mathcal{S} \mathfrak{F}$ that

$$
\int|f(x)-g(x)+h(x)| \phi d x \leqq r
$$

and

$$
\int\{g(x)+h(x)\} \phi d x \leqq \int|f(x)| \phi d x+r .
$$


4.8 Theorem. If $\mathfrak{F} \in \mathrm{bsc} \phi, \mathcal{S}=\operatorname{rlm} \phi, 0<r<\infty, f \in$ Summable $\phi$, then there are such members $g$ and $h$ of Crct $\mathcal{S} \mathfrak{F}$ that

$$
\operatorname{Alm} \phi x\{f(x)=g(x)-h(x)\}
$$

and

$$
\int\{g(x)+h(x)\} \phi d x \leqq \int|f(x)| \phi d x+r .
$$

Proof. Let us agree herein that

$$
n^{*}=E m \in \omega(m<n) .
$$

With the aid of 4.7, inductively define such a sequence $S$ of members of Crct $S F$ and such a sequence $T$ of members of Crct $S \mathfrak{F}$ that, for $n \in \omega$,

$$
\begin{gathered}
.1 \int\left|f(x)-\sum j \in n^{*} S_{j}(x)+\sum j \in n^{*} T_{j}(x)-S_{n}(x)+T_{n}(x)\right| \phi d x \\
\leqq r \cdot 2^{-(n+3)}
\end{gathered}
$$

and

$$
\begin{aligned}
& \int\left\{S_{n}(x)+T_{n}(x)\right\} \phi d x \\
& \quad \leqq \int\left|f(x)-\sum j \in n^{*} S_{j}(x)+\sum j \in n^{*} T_{j}(x)\right| \phi d x+r \cdot 2^{-(n+3)} .
\end{aligned}
$$

Now let $g$ and $h$ be such function on $S$ that for $x \in S$

$$
g(x)=\sum n \in \omega S_{n}(x)
$$

and

$$
h(x)=\sum n \in \omega T_{n}(x) .
$$

It is easily checked that

$$
g \in \operatorname{CrctS} \mathfrak{F}, \quad h \in \operatorname{Crct} S \mathfrak{F} .
$$

From .1 we see that if $n \in \omega$, then

$$
\begin{gathered}
\int \mid f(x)-\sum j \in(n+1)^{*} S_{j}(x)+\sum j \in \underset{(n+1)^{*} T_{j}(x) \mid \phi d x}{\leqq} \cdot 2^{-(n+3)} .
\end{gathered}
$$

From .2 we learn that

$$
\int\left\{S_{0}(x)+T_{0}(x)\right\} \phi d x \leqq \int|f(x)| \phi d x+r / 8,
$$


and from .2 and .3 we learn that if $n \in \omega$ then

$$
\begin{aligned}
\int\left\{S_{n+1}(x)+T_{n+1}(x)\right\} \phi d x & \leqq r \cdot 2^{-(n+8)}+r \cdot 2^{-(n+4)} \\
& \leqq r \cdot 2^{-(n+2)} .
\end{aligned}
$$

Thus

$$
\begin{aligned}
\int\{g(x)+ & h(x)\} \phi d x \\
& =\sum n \in \omega \int\left\{S_{n}(x)+T_{n}(x)\right\} \phi d x \\
& =\int\left\{S_{0}(x)+T_{0}(x)\right\} \phi d x+\sum n \in \omega \int\left\{S_{n+1}(x)+T_{n+1}(x)\right\} \phi d x \\
& \leqq \int|f(x)| \phi d x+r / 8+\sum n \in \omega r \cdot 2^{-(n+2)} \\
& =\int|f(x)| \phi d x+r / 8+r / 2 \\
& \leqq \int|f(x)| \phi d x+r<\infty
\end{aligned}
$$

Accordingly

$$
\operatorname{Alm} \phi x\{|f(x)|+|g(x)|+|h(x)|<\infty\}
$$

and from .3 and Fatou's Lemma it now follows that

$$
\int|f(x)-g(x)+h(x)| \phi d x=0 .
$$

Consequently

$$
\operatorname{Alm} \phi x\{f(x)=g(x)-h(x)\} .
$$

4.9 Theorem. If $\mathfrak{F} \in \mathrm{bsc} \phi, S=\operatorname{rlm} \phi, f \in$ Integrable $+\phi$, then there are such members $g$ and $h$ of Crct $S \mathfrak{F}$ that

$$
\operatorname{Alm} \phi x\{f(x)=g(x)-h(x)\}
$$

and

$$
\int h(x) \phi d x \leqq 1
$$

Proof. In some way ascertain first, as can clearly be done, such a sequence 
$S$ of members of Summable $+\phi$ that

$$
f(x)=\sum n \in \omega S_{n}(x) \quad \text { whenever } x \in \mathcal{S} .
$$

In accordance with 4.8 choose such a sequence $p$ of members of Crct $\delta \mathfrak{F}$ and such a sequence $q$ of members of Crct $\mathcal{F}$ that, for $n \in \omega$,

$$
\operatorname{Alm} \phi x\left\{S_{n}(x)=p_{n}(x)-q_{n}(x)\right\}
$$

and

$$
\int\left\{p_{n}(x)+q_{n}(x)\right\} \phi d x \leqq \int S_{n}(x) \phi d x+2^{-n} .
$$

Thus if $n \in \omega$, then:

$$
\begin{aligned}
\int p_{n}(x) \phi d x+\int q_{n}(x) \phi d x & \leqq \int S_{n}(x) \phi d x+2^{-n} \\
& =\int p_{n}(x) \phi d x-\int q_{n}(x) \phi d x+2^{-n} \\
2 \cdot \int q_{n}(x) \phi d x & \leqq 2^{-n} \\
\quad \int q_{n}(x) \phi d x & \leqq 2^{-(n+1)} .
\end{aligned}
$$

Now let $g$ and $h$ be such members of $\operatorname{Crct} \mathcal{S} \mathfrak{F}$ that, for $x \in \mathcal{S}$,

$$
g(x)=\sum n \in \omega p_{n}(x)
$$

and

$$
h(x)=\sum n \in \omega q_{n}(x) .
$$

From .1 we see that

$$
\int h(x) \phi d x \leqq \sum n \in \omega 2^{-(n+1)}=1 .
$$

Accordingly

$$
\operatorname{Alm} \phi x\{0 \leqq h(x)<\infty
$$

and

$$
\begin{aligned}
-\infty<g(x)-h(x) & =\sum n \in \omega p_{n}(x)-\sum n \in \omega q_{n}(x) \\
& \left.=\sum n \in \omega\left\{p_{n}(x)-q_{n}(x)\right\}=\sum n \in \omega S_{n}(x)=f(x)\right\} .
\end{aligned}
$$

Aided by $4.9,4.2 .1,4.2 .3$, and 4.2 .5 we arrive at 
4.10 Theorem. If $\mathfrak{F} \in \mathrm{bsc} \phi, \mathcal{S}=\operatorname{rlm} \phi, f \in \operatorname{Integrable} \phi$, then there are such members $g$ and $h$ of Crct $\mathcal{S} \mathfrak{F}$ that

$$
\operatorname{Alm} \phi x\{f(x)=g(x)-h(x)\}
$$

and

$$
\int f(x) \phi d x=\int g(x) \phi d x-\int h(x) \phi d x .
$$

5. General Fubini theory.

5.0 Definitions.

$.1 \mathrm{Mspr} \mu \nu \mathfrak{F}=\mathrm{E} \phi \in \mathrm{Msr}$ rct $\operatorname{rlm} \mu \operatorname{rlm} \nu[\mu \in$ Measure, $\nu \in$ Measure, $\mathfrak{F} \in$ bsc $\phi$, and

$$
\iint \operatorname{Cr}(x, y) A \mu d x \nu d y=\phi(A)=\iint \operatorname{Cr}(x, y) A \nu d y \mu d x
$$

for each $A \in \mathfrak{F}$.

In .1 above we made use of 4.5 .

We shall now employ 2.3.2.

.2 mblrct $\mu \nu=$ Ret $\mathrm{mbl} \mu \mathrm{mbl} \nu$.

.3 bscrct $\mu \nu=$ Ret $\mathrm{mbl}^{\prime} \mu \mathrm{mbl}^{\prime} \nu$.

.4 nil $\mu \nu=\mathrm{E} S \subset$ rct $\operatorname{rlm} \mu \operatorname{rlm} \nu[\mu \in$ Measure, $\nu \in$ Measure,

$$
\iint \operatorname{Cr}(x, y) S \mu d x \nu d y=0=\iint \operatorname{Cr}(x, y) \operatorname{S\nu dy\mu dx]} .
$$

.5 bace $\mu \nu=$ bscrct $\mu \nu \cup$ nil $\mu \nu$.

In connection with the preliminary basic estimate given in .6 below the reader may prefer to notice 5.5.2 now instead of later.

.6 bs $\mu \nu=$ the function $g$ on bace $\mu \nu$ such that

$$
g(S)=\iint \operatorname{Cr}(x, y) S \mu d x \nu d y
$$

whenever $S \in$ bace $\mu \nu$.

$.7 \mathrm{mspr} \mu \nu=\operatorname{mss}(\mathrm{bs} \mu \nu)(\operatorname{rct} \operatorname{rlm} \mu \operatorname{rlm} \nu)($ bace $\mu \nu)$.

.8 Fubini $\mu \nu=\mathrm{E} \phi \in \mathrm{Msr}$ rct $\operatorname{rlm} \mu \operatorname{rlm} \nu[\mu \in$ Measure, $\nu \in$ Measure, bscrct $\mu \nu \subset \mathrm{mbl}^{\prime} \phi$,

$$
\iint f(x, y) \mu d x \nu d y=\int f(z) \phi d z=\iint f(x, y) \nu d y \mu d x
$$

whenever $f \in$ Integrable $\phi]$.

Theorems 5.1-5.3 below were given by A. P. Morse in his 1949-1950 Lectures on Real Variables at the University of California. 
5.1 TheOREM. If $\phi \in \operatorname{Mspr} \mu \nu \mathfrak{F}, \mathcal{S}=\operatorname{rlm} \phi$ and $f \in \operatorname{Crct} \mathcal{S} \mathfrak{F}$ then

$$
\iint f(x, y) \mu d x \nu d y=\int f(z) \phi d z=\iint f(x, y) \nu d y \mu d x .
$$

Hint. After recalling 4.5.3 choose such a $\lambda$ from the 4.5.1 family cps $\mathfrak{F}$ and such a countable subfamily $\mathcal{S}$ of $\mathfrak{F}$ that $f(z)=\sum \beta \in \mathcal{S}\{\lambda(\beta) \cdot \mathrm{Cr} z \beta\}$ for each $z \in \mathcal{S}$. Now use 5.0.1, 4.5.4, the fact that $\leftrightarrow \subset \mathfrak{F} \subset \mathrm{cmbl} \phi, 4.4$, and integration by summation.

5.2 Theorem. If $\phi \in \operatorname{Mspr} \mu \nu \mathfrak{F}$ and $\operatorname{Alm} \phi z\{f(z)=g(z)\}$ then

$$
\iint f(x, y) \mu d x \nu d y=\iint g(x, y) \mu d x \nu d y
$$

and

$$
\iint f(x, y) \nu d y \mu d x=\iint g(x, y) \nu d y \mu d x .
$$

Proof. Let $S=\operatorname{rlm} \phi, A=\mathrm{Ez} \in \mathcal{S}[f(z) \neq g(z)]$. In accordance with 4.6 choose a sequence $h$ of members of $\operatorname{Crct} S \mathfrak{F}$ so that for each $n \in \omega$

$$
\operatorname{Cr} z A \leqq h_{n}(z) \quad \text { for each } z \in \mathcal{S}
$$

and

.1

$$
\int h_{n}(z) \phi d z \leqq 2^{-n}
$$

Let $h^{\prime}$ be such a function on $S$ that

$$
h^{\prime}(z)=\lim _{n} \inf h_{n}(z)
$$

whenever $z \in \mathcal{S}$.

Using .1, 5.1 and Fatou's lemma we see

$$
\begin{aligned}
0 & =\underset{n}{\liminf } \int h_{n}(z) \phi d z \\
& =\liminf _{n} \iint h_{n}(x, y) \mu d x \nu d y \\
& \geqq \int \liminf _{n} \int h_{n}(x, y) \mu d x \nu d y \\
& \geqq \iint h^{\prime}(x, y) \mu d x \nu d y \\
& \geqq 0 .
\end{aligned}
$$


Moreover, if $x \in \operatorname{rlm} \mu$ and $y \in \operatorname{rlm} \nu$ then $\operatorname{Cr}(x, y) A \leqq h^{\prime}(x, y)$. Thus

$$
\operatorname{Alm} \nu y\left(\int h^{\prime}(x, y) \mu d x=0\right) \text {, }
$$

Alm $\nu y \operatorname{Alm} \mu x\left(h^{\prime}(x, y)=0\right)$,

$\operatorname{Alm} \nu y \operatorname{Alm} \mu x(\operatorname{Cr}(x, y) A=0)$,

$\operatorname{Alm} \nu y \operatorname{Alm} \mu x(f(x, y)=g(x, y))$,

$$
\begin{aligned}
\operatorname{Alm} \nu y & \left(\int f(x, y) \mu d x=\int g(x, y) \mu d x\right), \\
& \iint f(x, y) \mu d x \nu d y=\iint g(x, y) \mu d x \nu d y .
\end{aligned}
$$

Similarly $\iint f(x, y) \nu d y \mu d x=\iint g(x, y) \nu d y \mu d x$.

5.3 Theorem. If $\phi \in$ Mspr $\mu \nu \mathfrak{F}$ and $f \in$ Integrable $\phi$ then

$$
\iint f(x, y) \mu d x \nu d y=\int f(z) \phi d z=\iint f(x, y) \nu d y \mu d x .
$$

Proof. Let $\delta=\operatorname{rlm} \phi$ and use 4.10 to secure such members $g$ and $h$ of Crct $\mathcal{S} F$ that

$$
\operatorname{Alm} \phi z(f(z)=g(z)-h(z))
$$

and

$$
\int f(z) \phi d z=\int g(z) \phi d z-\int h(z) \phi d z .
$$

Thus with the help of $5.1,4.6 .1,5.2$ and the fact that $f \in$ Integrable $\phi$ we infer

$$
\begin{aligned}
\int f(z) \phi d z & =\int g(z) \phi d z-\int h(z) \phi d z \\
& =\iint g(x, y) \mu d x \nu d y-\iint h(x, y) \mu d x \nu d y \\
& =\int\left[\int g(x, y) \mu d x-\int h(x, y) \mu d x\right] \nu d y \\
& =\iint[g(x, y)-h(x, y)]_{\mu d x \nu d y} \\
& =\iint f(x, y) \mu d x \nu d y .
\end{aligned}
$$


Similarly

$$
\int f(z) \phi d z=\iint f(x, y) \nu d y \mu d x
$$

and the desired conclusion is at hand.

5.4 Remark. We feel that 5.3 is not only of interest in itself but also of real use in checking various Fubini Theorems. In the present paper we shall use 5.3 four times. We apply it first in the proof of the pivotal 5.11 and later in the proofs of 7.11, 7.12 and 7.13.

5.5 Theorems.

.1 If $A \in \operatorname{mbl} \mu, B \in \operatorname{mbl} \nu, S=\operatorname{rct} A B$,

$$
r=\iint \operatorname{Cr}(x, y) S \mu d x \nu d y, \quad s=\iint \operatorname{Cr}(x, y) S \nu d y \mu d x,
$$

and either

$$
r<\infty, \text { or } \mu(A) \cdot \nu(B)<\infty \text {, or } s<\infty \text {, }
$$

then

$$
r=\mu(A) \cdot \nu(B)=s
$$

.2 If $S \in$ bace $\mu \nu$ then

$$
0 \leqq \iint \operatorname{Cr}(x, y) S \mu d x \nu d y=\iint \operatorname{Cr}(x, y) S \nu d y \mu d x<\infty .
$$

.3 If $S \in$ bace $\mu \nu$ and $g=$ bs $\mu \nu$ then

$$
0 \leqq g(S)=\iint \operatorname{Cr}(x, y) S \mu d x \nu d y<\infty .
$$

.4 If $\mu \in$ Measure and $\nu \in$ Measure then

$$
\operatorname{mspr} \mu \nu \in \mathrm{Msr} \operatorname{rct} \operatorname{rlm} \mu \operatorname{rlm} \nu \text {. }
$$

.5 If $A \in \mathrm{mbl}^{\prime} \mu, B \in \mathrm{mbl}^{\prime} \nu, g=\mathrm{bs} \mu \nu$ then

$$
g(\operatorname{rct} A B)=\mu(A) \cdot \nu(B) .
$$

.6 If $\alpha \subset \beta \in$ nil $\mu \nu$ and $g=b s \mu \nu$ then

$$
\alpha \in \text { nil } \mu \nu \text { and } g(\alpha)=0 .
$$

.7 If $S \in$ mblrct $\mu \nu$ and $\beta \in$ bace $\mu \nu$ then $\beta S \in$ bace $\mu \nu$.

5.6 TheOREM. mblrct $\mu \nu \subset \mathrm{mbl} \mathrm{mspr} \mu \nu$.

Proof. We assume 


$$
\phi=\operatorname{mspr} \mu \nu, \quad S=\operatorname{rlm} \phi, \quad S \in \operatorname{mblrct} \mu \nu,
$$

and complete the proof by showing

$$
S \in \operatorname{mbl} \phi .
$$

Suppose that

$$
\begin{gathered}
A \in \operatorname{mbl} \mu, \quad B \in \operatorname{mbl} \nu \\
S=\operatorname{rct} A B, \quad X=\operatorname{rlm} \mu, \quad \Upsilon=\operatorname{rlm} \nu, \\
S^{\prime}=\operatorname{rct}(X \sim A) B, \quad S^{\prime \prime}=\operatorname{rct} X(\Upsilon \sim B),
\end{gathered}
$$

and that

$$
g=\mathrm{bs} \mu \nu .
$$

Noting that $S=S \cup S^{\prime} \cup S^{\prime \prime}$ and hence that

$.1 \delta \sim S \subset S^{\prime} \cup S^{\prime \prime}$

we divide the remainder of the proof into three parts.

PART I. If $\beta \in$ bscrct $\mu \nu$ then

$$
g(\beta)=g(\beta S)+g\left(\beta S^{\prime}\right)+g\left(\beta S^{\prime \prime}\right) .
$$

Proof. Suppose

$$
\begin{gathered}
a \in \mathrm{mbl}^{\prime} \mu, \quad b \in \mathrm{mbl}^{\prime} \nu, \\
\beta=\operatorname{rct} a b .
\end{gathered}
$$

Clearly,

$$
\begin{aligned}
\beta S=\operatorname{rct}(a A)(b B), \quad \beta S^{\prime} & =\operatorname{rct}(a \sim A)(b B), \\
\beta S^{\prime \prime}=\operatorname{rct} a(b & \sim B) .
\end{aligned}
$$

Thus, in the light of 5.5.5,

$$
\begin{gathered}
g(\beta S)+g\left(\beta S^{\prime}\right)+g\left(\beta S^{\prime \prime}\right)=\mu(a A) \cdot \nu(b B)+\mu(a \sim A) \cdot \nu(b B)+\mu(a) \cdot \nu(b \sim B) \\
=\mu(a) \cdot \nu(b B)+\mu(a) \cdot \nu(b \sim B) \\
=\mu(a) \cdot \nu(b)=g(\beta) .
\end{gathered}
$$

PART II. If $\beta \in$ bace $\mu \nu$ then

$$
g(\beta)=g(\beta S)+g\left(\beta S^{\prime}\right)+g\left(\beta S^{\prime \prime}\right) .
$$

Proof. If $\beta \in$ bscrct $\mu \nu$ then the desired conclusion is a consequence of Part I. If $\beta \in$ nil $\mu \nu$ then it is a consequence of 5.5.6.

PART III. $S \in \mathrm{mbl} \phi$.

Proof. Suppose $r>0$ and $\phi(T)<\infty$. Choose such a countable subfamily $\mathfrak{F}$ of bace $\mu \nu$ that 


$$
T \subset \sigma \mathfrak{F} \text { and } \sum \beta \in \mathfrak{F} g(\beta) \leqq \phi(T)+r .
$$

Using .1, 5.5.7, and Part II we infer

$$
\begin{aligned}
\phi(T) & \leqq \phi(T S)+\phi(T \sim S) \\
& \leqq \phi(T S)+\phi\left(T S^{\prime}\right)+\phi\left(T S^{\prime \prime}\right) \\
& \leqq \sum \beta \in \mathfrak{F} \phi(\beta S)+\sum \beta \in \mathfrak{F} \phi\left(\beta S^{\prime}\right)+\sum \beta \in \mathfrak{F} \phi\left(\beta S^{\prime \prime}\right) \\
& \leqq \sum \beta \in \mathfrak{F} g(\beta S)+\sum \beta \in \mathfrak{F} g\left(\beta S^{\prime}\right)+\sum \beta \in \mathfrak{F} g\left(\beta S^{\prime \prime}\right) \\
& =\sum \beta \in \mathfrak{F}\left\{g(\beta S)+g\left(\beta S^{\prime}\right)+g\left(\beta S^{\prime \prime}\right)\right\} \\
& =\sum \beta \in \mathfrak{F} g(\beta) \leqq \phi(T)+r .
\end{aligned}
$$

The arbitrary nature of $r$ assures us

$$
\phi(T)=\phi(T S)+\phi(T \sim S) .
$$

5.7 Lemma. If $\phi=\operatorname{mspr} \mu \nu$ and $N \in$ nil $\mu \nu$ then $\phi(N)=0$.

5.8 Theorem. bace $\mu \nu \subset \mathrm{mbl} \mathrm{mspr} \mu \nu$.

5.9 Theorem. If $S \in$ bace $\mu \nu, \phi=\operatorname{mspr} \mu \nu$ and $g=\mathrm{bs} \mu \nu$ then

$$
\phi(S)=g(S)<\infty .
$$

Proof. From 5.5.3 we know $g(S)<\infty$.

Since $\phi(S) \leqq g(S)$ the desired conclusion now follows from the Statement. If $r>0$, then $g(S) \leqq \phi(S)+r$.

Proof. Choose such a countable subfamily $\mathfrak{F}$ of bace $\mu \nu$ that $S \subset \sigma \mathfrak{F}$ and $\sum \beta \in \mathfrak{F} g(\beta) \leqq \phi(S)+r$. Observe that, for each $z$,

$$
0 \leqq \operatorname{Cr} z S \leqq \sum \beta \in \mathfrak{F} \operatorname{Cr} z \beta
$$

and hence that

$$
\begin{aligned}
\phi(S)+r \geqq \sum \beta \in \mathfrak{F} g(\beta) & =\sum \beta \in \mathfrak{F} \iint \mathrm{Cr}(x, y) \beta \mu d x \nu d y \\
& =\int \sum \beta \in \mathfrak{F} \int \operatorname{Cr}(x, y) \beta \mu d x \nu d y \\
& =\iint \sum \beta \in \mathfrak{F r}(x, y) \beta \mu d x \nu d y \\
& \geqq \iint \operatorname{Cr}(x, y) S \mu d x \nu d y \\
& =g(S) .
\end{aligned}
$$

5.10 ThEOREMS.

.1 If $\mu \in$ Measure, $\nu \in$ Measure, 


$$
\phi=\operatorname{mspr} \mu \nu, \quad \mathfrak{F}=\text { bace } \mu \nu,
$$

then

$$
\mathfrak{F} \subset \mathrm{mbl}^{\prime} \phi \text { and } \mathfrak{F} \in \mathrm{bsc} \phi .
$$

Proof. Use 5.8, 5.9, 5.0.7 and 4.5.4.

.2 If $S \in$ bace $\mu \nu$ and $\phi=\operatorname{mspr} \mu \nu$ then

$$
\iint \operatorname{Cr}(x, y) S \mu d x \nu d y=\phi(S)=\iint \operatorname{Cr}(x, y) S \nu d y \mu d x .
$$

Proof. Use 5.9, 5.5.3, and 5.5.2.

.3 If $\mu \in$ Measure, $\nu \in$ Measure, $\phi=\operatorname{mspr} \mu \nu$, and $\mathfrak{F}=$ bace $\mu \nu$, then

$$
\phi \in \operatorname{Mspr} \mu \nu \mathfrak{} .
$$

Proof. Use .1, .2, and 5.5.4.

5.11 Theorem. If $\mu \in$ Measure, $\nu \in$ Measure, $\phi=\operatorname{mspr} \mu \nu$ then:

$.1 \mathrm{mblrct} \mu \nu \subset \mathrm{mbl} \phi$;

.2 if $N \in$ nil $\mu \nu$ then $\phi(N)=0$;

$.3 \phi \in$ Fubini $\mu \nu$.

Proof. Use 5.6, 5.7, 5.10.3, 5.3 and the fact that 5.10.1 assures us

$$
\text { bscrct } \mu \nu \subset \mathrm{mbl}^{\prime} \phi \text {. }
$$

5.12 Remark. If $\mu \in$ Measure, $\nu \in$ Measure,

$$
\phi=\operatorname{mspr} \mu \nu,
$$

and

$$
\psi=\operatorname{mss}(\mathrm{bs} \mu \nu)(\operatorname{rct} \operatorname{rlm} \mu \operatorname{rlm} \nu)(\text { bscrct } \mu \nu)
$$

then $\psi$ is the classical product measure and the distinction between it and $\phi$ is, in essence, that

$$
\begin{aligned}
& \psi \in \operatorname{Mspr} \mu \nu \text { bscrct } \mu \nu, \\
& \phi \in \operatorname{Mspr} \mu \nu \text { bace } \mu \nu, \\
& \text { bace } \mu \nu=\text { bscrct } \mu \nu \cup \text { nil } \mu \nu, \\
& \text { mbl } \psi \subset \text { mbl } \phi .
\end{aligned}
$$

It is just this difference which makes $\phi$ so much more valuable than $\psi$ when topological considerations are involved.

We now examine some other aspects of our product measure.

5.13 Theorem. If $A \in \mathrm{mbl} \mu, B \in \mathrm{mbl} \nu$, and $\phi=\operatorname{mspr} \mu \nu$, then 


$$
\phi(\operatorname{rct} A B)=\mu(A) \cdot \nu(B) .
$$

Proof. Let $S=\operatorname{rct} A B$ and infer the desired conclusion from Parts I and II below.

PART I. If $\phi(S)<\infty$ then

$$
\phi(S)=\mu(A) \cdot \nu(B) .
$$

Proof. Use 5.11 and 5.5.1.

PART II. If $\mu(A) \cdot \nu(B)<\infty$ then

$$
\phi(S)=\mu(A) \cdot \nu(B) .
$$

Proof. If $\mu(A) \cdot \nu(B)=0$ then, because of 5.5.1,

$$
S \in \operatorname{nil} \mu \nu \subset \text { bace } \mu \nu \text {. }
$$

On the other hand if $0<\mu(A) \cdot \nu(B)<\infty$ then

$$
S \in \text { bscrct } \mu \nu \subset \text { bace } \mu \nu \text {. }
$$

Consequently $S \in$ bace $\mu \nu$, and from 5.10.2 and 5.5.1, it now follows that $\phi(S)=\mu(A) \cdot \nu(B)$.

5.14 Theorem. If $\mu \in$ Measure, $\nu \in$ Measure, $\phi=\operatorname{mspr} \mu \nu, \phi(T)<\infty$, and $r>0$ then there is such a countable subfamily $\mathfrak{F}$ of bscrct $\mu \nu$ and such a member $T^{\prime}$ of bscrct $\mu \nu$ that:

$.1 \phi(T \sim \sigma \mathfrak{F})=0 ; \quad \sum S \in \mathfrak{F} \phi(S) \leqq \phi(T)+r ;$

$.2 \phi\left(T \sim T^{\prime}\right) \leqq r$.

5.15 Theorem. If $A \in \mathrm{mbl} \mu$ and $B \in \mathrm{mbl} \nu$ then

$$
\text { sct }(\operatorname{mspr} \mu \nu)(\operatorname{rct} A B)=\operatorname{mspr}(\operatorname{sct} \mu A)(\operatorname{sct} \nu B) \text {. }
$$

By following an alternative course indicated in the proof of 7.5 it is quite feasible to avoid all use of 5.16 and 5.17 .

5.16 Definition. inv $R=\mathrm{E} z[z$ is of the form $(y, x)$ where $(x, y) \in R]$.

5.17 Theorem. If $\mu \in$ Measure, $\nu \in$ Measure, $\phi=\operatorname{mspr} \mu \nu, \phi^{\prime}=\operatorname{mspr} \nu \mu$, then

$$
\operatorname{dmn} \phi^{\prime}=\operatorname{inv} \mathrm{dmn} \phi
$$

and

$$
\phi^{\prime}(\operatorname{inv} \beta)=\phi(\beta) \quad \text { whenever } \beta \in \operatorname{dmn} \phi .
$$

6. Topological measures.

6.0 DEFINITIONS.

.1 topology $=\mathrm{E} \mathfrak{T}[\mathfrak{T}$ is such a family of sets that:

$$
\begin{array}{cr}
\sigma \mathfrak{F} \in \mathfrak{T} & \text { whenever } \mathfrak{F} \subset \mathfrak{T} ; \\
\alpha \cap \beta \in \mathfrak{T} & \text { whenever } \alpha \in \mathfrak{T} \text { and } \beta \in \mathfrak{T}] .
\end{array}
$$


If, for example, $\mathfrak{T}$ is the family of all open subsets of a metric space, then $\mathfrak{T} \in$ topology.

$.2 \mathfrak{T}$ topologizes $S$ if and only if $\mathfrak{T} \in$ topology and $S=\sigma \mathfrak{T}$.

.3 Closed $\mathfrak{T}=\mathrm{E} C[\mathfrak{T} \in$ topology and $C=\sigma \mathfrak{T} \sim A$ for some $A \in \mathfrak{T}]$.

.4 Compact $\mathfrak{T}=\mathrm{E} C[\mathfrak{T} \in$ topology and corresponding to each subfamily $\mathfrak{F}$ of $\mathfrak{T}$ for which $C \subset \sigma \mathfrak{F}$ there exists a finite subfamily $\mathfrak{E}$ of $\mathfrak{F}$ such that $C \subset \sigma \mathfrak{Q}]$.

We now use 2.1.4.

.5 Closure $\mathfrak{T} A=\pi \mathrm{E} B(A \subset B \in$ Closed $\mathfrak{T})$.

.6 regulartopology $=\mathrm{E} \mathfrak{T} \in$ topology [corresponding to each $A \in \mathfrak{T}$ and each $x \in A$ there is a $B \in \mathfrak{T}$ such that $x \in B$ and Closure $\mathfrak{T} B \subset A]$.

.7 Lindelöftopology $=\mathrm{E} \mathfrak{T} \in$ topology [corresponding to each $\mathfrak{F} \subset \mathfrak{T}$ for which $\sigma \mathfrak{F}=\sigma \mathfrak{T}$ there is such a countable subfamily $\mathbb{S}$ of $\mathfrak{F}$ that $\sigma \mathfrak{S}=\sigma \mathfrak{T}$ ].

6.1 Definition. sp' $\phi A=\mathrm{E} B C \operatorname{rlm} \phi[\phi(A \sim B)=0]$.

In connection with $6.2 .3-6.2 .5$ below we suggest that the symbols

$$
\text { 'Oh', 'Bh', 'Ck' }
$$

be respectively read as one would

'Openhull', 'Borelhull', 'Closedkernel'.

Before pursuing 6.2 and 6.3 the reader may wish to recall Definitions 3.3, 3.5, and 3.6.

6.2 Definitions.

.1 Core $\mathfrak{T}=$ Mass Closed $\mathfrak{T}$.

$.2 \mathrm{Oh}^{\prime} \mathfrak{T}=\mathrm{E} \phi \in \mathrm{Msr} \sigma \mathfrak{T}[\mathfrak{T} \in$ topology and for each $A \subset \sigma \mathfrak{T}$

$$
\left.\phi(A)=\inf \alpha \in \mathfrak{T} \cap \mathrm{sp}^{\prime} \phi A \phi(\alpha)\right] .
$$

.3 Oh $\mathfrak{T}=\mathrm{E} \phi \in \mathrm{Msr} \sigma \mathfrak{T}[\mathfrak{T} \in$ topology and for each $A \subset \sigma \mathfrak{T}$

$$
\phi(A)=\inf \alpha \in \mathfrak{T} \cap \operatorname{sp} A \phi(\alpha)] .
$$

.4 Bh $\mathfrak{T}=$ Hull Borel $\mathfrak{T}$.

$.5 \mathrm{Ck} \mathfrak{T}=\mathrm{E} \phi \in \mathrm{Msr} \sigma \mathfrak{T}[\mathfrak{T} \in$ topology and for each $A \in \mathfrak{T}$

$$
\left.\phi(A)=\sup \beta \in \text { Closed } \mathfrak{T} \cap \mathrm{dmn}^{\prime} \phi \cap \operatorname{sb} A \phi(\beta)\right] .
$$

From 3.7.1, 3.15, and 3.16 we infer at once the

\subsection{Theorems.}

.1 If $\mathfrak{T} \in$ Topology then

Borel $\mathfrak{T}=$ Borel Closed $\mathfrak{T} \subset$ Bore Closed $\mathfrak{T}$.

.2 If $\phi \in$ Core $\mathfrak{T}$ then

Bore $\mathfrak{T} \cap d \mathrm{mn}^{\prime} \phi \subset$ prxn Closed $\mathfrak{T}$.

.3 If $\phi \in$ Core $\mathfrak{T} \cap$ Hull Bore Closed $\mathfrak{T}$ then 


$$
\operatorname{mbl}^{\prime} \phi \subset \operatorname{prxn} \phi \text { Closed } \mathfrak{T} \text {. }
$$

6.4 Definitions.

.1 Lind $\mathfrak{T}=\mathrm{E} \phi \in \mathrm{Msr} \sigma \mathfrak{T}[\mathfrak{T} \in$ topology, corresponding to each $\psi \in \operatorname{sms} \phi$ and each $\mathfrak{F} \subset \mathfrak{T}$ for which $\sigma \mathfrak{F}=\sigma \mathfrak{T}$ there is a countable subfamily $\mathbb{H}$ of $\mathfrak{F}$ for which

$$
\psi(\sigma \mathfrak{T} \sim \sigma(\mathfrak{S})=0] .
$$

.2 Clin $\mathfrak{T}=$ Core $\mathfrak{I} \cap$ Lind $\mathfrak{T}$.

.3 Radonmeasure $\mathfrak{I}=\mathrm{E} \phi \in \mathrm{Clin} \mathfrak{I} \cap \mathrm{Oh} \mathfrak{T} \cap \mathrm{Ck} \mathfrak{T}$

$[\mathfrak{T} \in$ locallycompactregulartopology and

$$
\text { Compact } \left.\mathfrak{T} \subset \mathrm{dmn}^{\prime} \phi\right] \text {. }
$$

In connection with 6.4 .1 we give 6.5 below.

6.5 Theorems.

.1 If $\mathfrak{T} \in$ topology, $\phi \in \mathrm{Msr} \sigma \mathfrak{T}$, and corresponding to each $\mathfrak{F} \subset \mathfrak{T}$ for which $\sigma \mathfrak{F}=\sigma \mathfrak{T}$ there is a countable subfamily $\mathbb{B}$ of $\mathfrak{F}$ for which

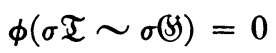

then

$$
\phi \in \operatorname{Lind} \mathfrak{T} .
$$

.2 If $\mathfrak{T} \in$ Lindelöftopology then

$$
\text { Lind } \mathfrak{T}=\text { Msr } \sigma \mathfrak{T} .
$$

In connection with 6.4 .3 one can see that

$$
\phi \in \text { Radonmeasure } \mathfrak{T}
$$

if and only if:

$$
\begin{aligned}
& \mathfrak{T} \in \text { locallycompactregulartopology; } \\
& \phi \in \mathrm{Oh} \mathfrak{T} ; \quad \mathfrak{T} \subset \mathrm{mbl} \phi ; \\
& \text { Compact } \mathfrak{T} \subset \mathrm{dmn}^{\prime} \phi ;
\end{aligned}
$$

for each $A \in \mathfrak{T}$

$$
\phi(A)=\sup \beta \in \text { Compact } \mathfrak{T} \cap \operatorname{sb} A \phi(\beta) .
$$

6.6 REMARK. The topological measures of particular interest to us in the sequel are

$$
\begin{array}{ll}
\text { Clin } \mathfrak{T}, & \text { Ck } \mathfrak{T} \text { Clin } \mathfrak{T}, \\
\text { Bh } \mathfrak{T} \text { Clin } \mathfrak{T}, & \text { Oh } \mathfrak{T} \text { Clin } \mathfrak{T}, \\
\text { Oh } \mathfrak{T} \text { Ck } \mathfrak{T} \text { Clin } \mathfrak{T} .
\end{array}
$$


Of these Clin $\mathfrak{I}$ is very general. If $\mathfrak{T}$ is the Euclidean topology for the plane then: every Hausdorff dimensional measure belongs to Bh $\mathfrak{T}$ Clin $\mathfrak{T}$; in particular if $\phi$ is Carathéodory linear measure then

$$
\phi \in \mathrm{Bh} \mathfrak{T} \text { Clin } \mathfrak{T}, \quad \mathfrak{T} \operatorname{cmbl} \phi=\text { sng } 0,
$$

and hence $\phi$ does not belong to

\section{Oh $\mathfrak{T}$ Clin $\mathfrak{T}$.}

Still more special than

\section{Oh $\mathfrak{T}$ Ck $\mathfrak{T}$ Clin $\mathfrak{T}$}

is

\section{Radonmeasure $\mathfrak{T}$.}

From different points of view this last class has received much attention. From our point of view one interesting aspect of it is given without proof in

6.7 Remark. Suppose

$$
\mathfrak{I} \in \text { locallycompactregulartopology }
$$

and suppose $\mathbb{C}$ is the set of all those continuous functions which are on $\sigma \mathfrak{T}$ to the real finite numbers, and which vanish outside of some member of Compact $\mathfrak{T}$.

Next suppose $u$ is such a linear functional on (5 that $u(f) \geqq 0$ whenever $f$ is such a member of $(5$ that $f(x) \geqq 0$ whenever $x \in \sigma \mathfrak{T}$.

For each $\beta \in$ Compact $\mathfrak{I}$ let $\psi(\beta)=$ the infimum of numbers of the form $u(f)$ where $f$ is such a member of $\mathcal{E}$ that

$$
\text { Cr } x \beta \leqq f(x) \quad \text { whenever } x \in \sigma \mathfrak{T} .
$$

Now let $\phi$ be such a function on $\operatorname{sb} \sigma \mathfrak{T}$ that for $A \subset \sigma \mathfrak{T}$

$$
\phi(A)=\inf \alpha \in \mathfrak{T} \cap \operatorname{sp} A \sup \beta \in \text { Compact } \mathfrak{T} \cap \operatorname{sb} \alpha \psi(\beta) .
$$

Either directly or in keeping with pp. 231-249, Paul R. Halmos, Measure theory, New York, 1950, it can be checked that:

$$
\phi \in \text { Radonmeasure } \mathfrak{T} \text {; }
$$

$$
u(f)=\int f(x) \phi d x \quad \text { whenever } f \in \mathbb{E} ;
$$

if $\phi^{\prime} \in$ Radonmeasure $\mathfrak{T}$ and

$$
u(f)=\int f(x) \phi^{\prime} d x \quad \text { whenever } f \in \mathcal{E}
$$


then

$$
\phi^{\prime}=\phi .
$$

In Theorem 6.8 below we study the reaction of $\phi$ to the taming process 6.8.5.

6.8 ThEOREM. If

$.1 \phi \in \mathrm{Msr} \sigma \mathfrak{T}$,

$.2 \mathfrak{G C s b} \sigma \mathfrak{T}$,

$.3 \sigma \Re \in \mathfrak{S}$ whenever $\Re$ is a countable subfamily of $\mathfrak{S}$,

$.4 \beta \cap \alpha \in \mathfrak{S}$ whenever $\beta \in \mathfrak{T}$ and $\alpha \in \mathfrak{W}$,

then:

$.5 \psi=\operatorname{mss} \phi \sigma \mathfrak{T} \mathfrak{W}$,

$.6 \psi \in \mathrm{Msr} \sigma \mathfrak{T}$;

.7 if $A \subset \sigma \mathfrak{T}$ then $\phi(A) \leqq \psi(A)$;

.8 if $A \in \mathfrak{W}$ then $\phi(A)=\psi(A)$;

.9 if $A \subset \sigma \mathfrak{T}$ then $\psi(A)=\inf \alpha \in \mathfrak{B} \cap \operatorname{sp} A \psi(\alpha)$;

.10 if $\phi \in$ Core $\mathfrak{T}, A \in$ Bore Closed $\mathfrak{T}$ and $T \in \mathscr{S} \mathrm{dmn}^{\prime} \psi$, then

$$
\psi(T A)=\phi(T A) ;
$$

.11 if $\phi \in$ Core $\mathfrak{T}$ then $\psi \in$ Core $\mathfrak{T}$;

.12 if $\phi \in C$ lin $\mathfrak{T}$ then $\psi \in$ Clin $\mathfrak{T}$;

.13 if $\mathfrak{E}=\mathfrak{T}$ and $\phi \in \mathrm{Oh}^{\prime} \mathfrak{T} \cap \mathrm{Ck} \mathfrak{T}$ then $\psi \in \mathrm{Oh} \mathfrak{T} \cap \mathrm{Ck} \mathfrak{T}$.

Proof. Let $\delta=\sigma \mathfrak{T}$. That

$$
\psi \in \operatorname{Msr} \delta
$$

is a consequence of 3.2. Evidently, also

$$
\psi(A)=\inf \alpha \in \mathfrak{S} \cap \operatorname{sp} A \phi(\alpha)
$$

for each $A C S$.

Proof .7. Using (1)

$$
\psi(A)=\inf \alpha \in \mathfrak{S} \cap \operatorname{sp} A \phi(\alpha) \geqq \phi(A) .
$$

Proof .8. Using (1) and .7

$$
\begin{aligned}
\psi(A) & =\inf \alpha \in \mathfrak{S} \cap \operatorname{sp} A \phi(\alpha) \\
& \leqq \phi(A) \leqq \psi(A) .
\end{aligned}
$$

Hence $\psi(A)=\phi(A)$.

Proof .9. Using (1) and .8

$$
\begin{aligned}
\psi(A) & =\inf \alpha \in \mathfrak{S} \cap \operatorname{sp} A \phi(\alpha) \\
& =\inf \alpha \in \mathfrak{G} \cap \operatorname{sp} A \psi(\alpha) .
\end{aligned}
$$

Proof .10. Let $r>0, \theta=\operatorname{sct} \phi T$. Thus 


$$
\theta(S)=\phi(T) \leqq \psi(T)<\infty
$$

and by 3.5 .3

$$
\theta \in \text { Core } \mathfrak{T} \text {. }
$$

Now we use 6.3.2 and the fact that

$$
S \sim A \in \text { Bore Closed } \mathfrak{T}
$$

to secure such a member $C$ of Closed $\mathfrak{T}$ that $C \subset \mathcal{S} \sim A$ and

$$
\theta(S \sim A \sim C) \leqq r,
$$

and let

$$
\alpha=\mathrm{S} \sim C .
$$

Thus $A \subset \alpha \in \mathfrak{T}, T A \subset T \alpha \in \mathfrak{W}$,

$$
\begin{aligned}
\psi(T A) & =\inf \beta \in \mathfrak{G} \cap \operatorname{sp}(T A) \phi(\beta) \\
& \leqq \phi(T \alpha) \\
& \leqq \phi(T A)+\phi(T \alpha \sim A) \\
& =\phi(T A)+\theta(\alpha \sim A) \\
& =\phi(T A)+\theta(S \sim C \sim A) \\
& \leqq \phi(T A)+r .
\end{aligned}
$$

Because of this and .7,

$$
\phi(T A) \leqq \psi(T A) \leqq \phi(T A)+r . \quad \cdot
$$

Proof .11. Let $A \in \mathfrak{T}, \psi(T)<\infty, r>0$.

The desired conclusion will be at hand after we have verified that:

$$
\psi(T)+r \geqq \psi(T A)+\psi(T \sim A) ;
$$

for some $C \in$ Closed $\mathfrak{T} \cap s b A$

$$
\text { sct } \psi T(A \sim C) \leqq r .
$$

Use (1) to secure such a member $T^{\prime}$ of $\mathfrak{E}$ that $T \subset T^{\prime}$ and

$$
\phi\left(T^{\prime}\right) \leqq \psi(T)+r .
$$

Using the fact that $A \in \mathrm{mbl} \phi$ and .10 we infer

$$
\begin{aligned}
\psi(T)+r & \geqq \phi\left(T^{\prime}\right) \\
& =\phi\left(T^{\prime} A\right)+\phi\left(T^{\prime} \sim A\right) \\
& =\psi\left(T^{\prime} A\right)+\psi\left(T^{\prime} \sim A\right) \\
& \geqq \psi(T A)+\psi(T \sim A) .
\end{aligned}
$$


Now use the fact that $\phi \in$ Core $\mathfrak{I}$ to secure such a member $C$ of Closed $\mathfrak{T}$ that $C \subset A$ and

$$
\operatorname{sct} \phi T^{\prime}(A \sim C) \leqq r ;
$$

and, because of .10 , conclude

$$
\begin{aligned}
\operatorname{sct} \psi T(A \sim C) & \leqq \operatorname{sct} \psi T^{\prime}(A \sim C) \\
& =\psi\left[T^{\prime}(A \sim C)\right] \\
& =\phi\left[T^{\prime}(A \sim C)\right] \\
& =\operatorname{sct} \phi T^{\prime}(A \sim C) \\
& \leqq r .
\end{aligned}
$$

Proof .12. Suppose $\psi(T)<\infty, \mathfrak{F} \subset \mathfrak{T}, \mathcal{S}=\sigma \mathfrak{F}$. Use (1) to so choose $T^{\prime} \in \mathfrak{W}$ that $T \subset T^{\prime}$ and $\phi\left(T^{\prime}\right)<\infty$. Next use the fact that $\phi \in$ Lind $\mathfrak{I}$ to choose such a countable subfamily $(\mathcal{S}$ of $\mathfrak{F}$ that

$$
\operatorname{sct} \phi T^{\prime}(S \sim \sigma())=0 .
$$

Thus, because of .10

$$
\begin{aligned}
\operatorname{sct} \psi T(S \sim \sigma(S) & \leqq \operatorname{sct} \psi T^{\prime}(S \sim \sigma(S) \\
& =\psi\left[T^{\prime}(S \sim \sigma())\right] \\
& =\phi\left[T^{\prime}(S \sim \sigma(S)]\right. \\
& =\operatorname{sct} \phi T^{\prime}(S \sim \sigma(S) \\
& =0 .
\end{aligned}
$$

Consequently $\psi \in$ Lind $\mathfrak{T}$.

From this and .11 it follows that

$$
\psi \in \operatorname{Clin} \mathfrak{T} \text {. }
$$

Proof .13. Suppose $A \in \mathfrak{T}, r<\psi(A)$.

Use the fact that $\phi \in \mathrm{Ck} \mathfrak{T}$ to secure such a member $C$ of Closed $\mathfrak{T}$ that

$$
C \subset A \text { and } r<\phi(C)<\infty \text {. }
$$

Use the fact that $\phi \in \mathrm{Oh}^{\prime} \mathfrak{T}$ to obtain such a member $\alpha$ of $\mathfrak{I} \cap \mathrm{sp}^{\prime} \phi C$ that

$$
\phi(\alpha) \leqq \phi(C)+1 \text {. }
$$

Observe that $\phi(\alpha A) \geqq \phi(C)>r$.

Now use the fact that $\phi \in C k \mathfrak{I}$ to secure such a member $C^{\prime}$ of Closed $\mathfrak{T}$ that $C^{\prime} \subset \alpha A$ and

$$
\phi\left(C^{\prime}\right)>r .
$$

Thus $C^{\prime} \subset A$ and 


$$
\begin{aligned}
r<\phi\left(C^{\prime}\right) & \leqq \psi\left(C^{\prime}\right) \leqq \psi(\alpha) \\
& \leqq \phi(C)+1<\infty .
\end{aligned}
$$

Consequently $\psi \in \mathrm{Ck} \mathfrak{T}$.

Furthermore, by .9, for each $D \subset S$ we have

$$
\begin{aligned}
\psi(D) & =\inf \alpha \in \mathfrak{S} \cap \operatorname{sp} D \psi(\alpha) \\
& =\inf \alpha \in \mathfrak{T} \cap \operatorname{sp} D \psi(\alpha),
\end{aligned}
$$

and thus

$$
\psi \in \mathrm{Oh} \mathfrak{T} .
$$

Locally null sets are now examined briefly.

6.9 Definition. null $\mathfrak{T} \phi A=\mathrm{E} \beta$ [Corresponding to each $x \in \beta$ there is an $\alpha \in \mathfrak{T}$ such that $x \in \alpha$ and $\phi(\alpha A)=0]$.

In 6.10 we see that null $\mathfrak{T} \phi A$ is hereditary.

6.10 Theorem. If $B^{\prime} \subset B \in$ null $\mathfrak{T} \phi A$ then $B^{\prime} \in$ null $\mathfrak{T} \phi A$.

6.11 Theorem. If $\phi \in$ Lind $\mathfrak{I}$ and

$$
C \in \text { Closed } \mathfrak{T} \cap \mathrm{dmn}^{\prime} \phi \cap \text { null } \mathfrak{T} \phi A
$$

then

$$
\phi(A C)=0 .
$$

Proof. Let $\boldsymbol{S}=\sigma \mathfrak{T}$,

$$
\psi=\operatorname{sct} \phi(A C), \quad \mathfrak{F}=\mathrm{E} \alpha \in \mathfrak{T}[\psi(\alpha)=0]
$$

and note that $\psi \in$ sms $\phi$.

We use the fact that

$$
C \in \text { Closed } \mathfrak{T} \cap \text { null } \mathfrak{T} \phi A
$$

to check that $\sigma \mathfrak{F}=\mathcal{S}$ and then the fact that $\phi \in$ Lind $\mathfrak{T}$ to secure such a countable subfamily $\mathbb{S}$ of $\mathfrak{F}$ that $\psi(\mathcal{S} \sim \sigma(\mathfrak{S})=0$. Accordingly $\psi(\sigma(\mathfrak{S})=0$ and

$$
\phi(A C)=\psi(\grave{\mathfrak{S}}) \leqq \psi(\sigma(\mathfrak{S})+\psi(\mathcal{S} \sim \sigma(\mathfrak{S})=0+0=0 .
$$

6.12 Theorem. If $\phi \in$ Clin $\mathfrak{T}$ and

$$
D \in \mathfrak{I} \cap \mathrm{dmn}^{\prime} \phi \cap \text { null } \mathfrak{T} \phi A
$$

then

$$
\phi(A D)=0 .
$$

Proof. Use the facts that $\phi \in$ Core $\mathfrak{I}$ and $\phi(D)<\infty$ to secure such a sequence $c$ that

$$
c_{n} \in \text { Closed } \mathfrak{I}, \quad c_{n} \subset D,
$$




$$
\phi\left(D \sim c_{n}\right) \leqq 2^{-n},
$$

for each $n \in \omega$.

Applying 6.10 and 6.11 we have for each $n \in \omega$,

$$
\begin{aligned}
\phi(A D) & \leqq \phi\left(A c_{n}\right)+\phi\left(D \sim c_{n}\right) \\
& \leqq 0+2^{-n} .
\end{aligned}
$$

Thus $\phi(A D)=0$.

We close this section with a short account of what does happen to Core $\mathfrak{T}$ and what might happen to Clin $\mathfrak{T}$ in the event $\mathfrak{T} \in$ metrictopology.

6.13 Definition. Md $\rho=\mathrm{E} \phi[\rho$ is a metric, $\phi \in \mathrm{Msr} \sigma$ open $\rho$,

$$
\phi(A \cup B)=\phi(A)+\phi(B)
$$

whenever

$$
\text { distance } \rho A B>0 \text { ]. }
$$

6.14 THEOREM. If $\rho$ is a metric and $\mathfrak{T}=$ open $\rho$ then

$$
\operatorname{Md} \rho=\text { Core } \mathfrak{T} \text {. }
$$

6.15 THEOREM. If $\rho$ is a separable metric and $\mathfrak{T}=$ open $\rho$ then

$$
\text { Md } \rho=\text { Clin } \mathfrak{T} \text {. }
$$

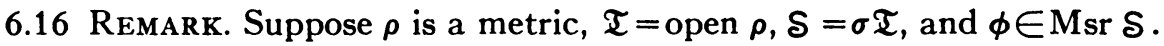
It is easy to verify that

$$
\phi \in \text { Lind } \mathfrak{T}
$$

if and only if :

(1) for each $\psi \in$ sms $\phi$, there exists $N \subset \mathcal{S}$ such that $\mathcal{S} \sim N$ is separable $\rho$ and $\psi(N)=0$.

Marczewski and Sikorski have shown $\left({ }^{2}\right)$ that if $\mathfrak{T} \subset \mathrm{mbl} \phi$ and if the separability character $\left({ }^{3}\right)$ of $\rho$ has measure zero $\left({ }^{2}\right)$ then the condition (1) holds.

S. Ulam has shown( $\left.{ }^{4}\right)$ that every cardinal number less than the first weakly inaccessible cardinal $\left({ }^{5}\right)$ has measure zero $\left({ }^{2}\right)$.

Thus we have the following theorem.

6.17 THEOREM. If $\rho$ is a metric, $\mathfrak{T}=$ open $\rho$, and the separability character $\left({ }^{3}\right)$ of $\rho$ is less than the first weakly inaccessible cardinal $\left({ }^{5}\right)$, then

(2) Marczewski and Sikorski, Measures in non-separable metric spaces, Colloquium Math. vol. 1 (1948) pp. 133-139.

(3) The smallest cardinal of the form, The power of $A$, where closure $\rho A=S$.

(4) S. Ulam, Zur Masstheorie in der allgemeinen Mengenlehre, Fund. Math. vol. 16 (1930) pp. 140-150. 69.

(5) See. A. Tarski, Über unerreichbare Kardinalzahlen, Fund. Math. vol. 30 (1938) pp. 68- 
$\operatorname{Md} \rho=\operatorname{Clin} \mathfrak{T}$.

The separability character of $\rho$ is, of course, not larger than the power of $\sigma \mathfrak{T}$.

7. Topological Fubini theory. We use 2.3.2 in 7.0.

7.0 Definition. $\mathfrak{M} \square \mathfrak{N}=$ The topological product of $\mathfrak{M}$ and $\mathfrak{N}$

$$
=\mathrm{E} \alpha(\alpha=\sigma \mathfrak{F} \text { for some } \mathfrak{F} \subset \text { Rct } \mathfrak{M} \mathfrak{R}) \text {. }
$$

It is well known and easy to see that if $\mathfrak{M} \in$ topology and $\mathfrak{R} \in$ topology then $\mathfrak{M} \square \mathfrak{R} \in$ topology and

$$
\sigma(\mathfrak{M} \square \mathfrak{R})=\operatorname{rct} \sigma \mathfrak{M} \sigma \mathfrak{R} .
$$

Theorem 7.7 below and 5.11 above allow wide latitude in interchanging order of integration. In other situations, however, one might prefer a product measure which more faithfully reflects the properties of the topological measures given in advance.

This leads us to 7.1.

7.1 Definitions.

.1 mspro $\mathfrak{M} \mathfrak{R} \mu \nu=$ mss mspr $\mu \nu \sigma(\mathfrak{M} \square \mathfrak{R})(\mathfrak{M} \square \mathfrak{N})$.

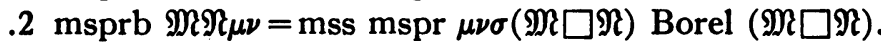

In 7.2 below we have vertical and horizontal slices in mind. We ourselves shall not use 7.2.2 but the reader may wish to if he chooses to pursue the alternative course indicated in the proof of 7.5.

7.2 Definitions.

.1 vs $S x=\mathrm{E} y[(x, y) \in S]$.

.2 hs $S y=\operatorname{Ex}[(x, y) \in S]$.

7.3 Definition. cbl $=$ the family of all countable sets.

7.4 Lemma. If $\mu \in \mathrm{Ck} \mathfrak{M}$ Clin $\mathfrak{M}, \nu \in \mathrm{Ck} \mathfrak{R}$ Clin $\mathfrak{N}, \phi=\mathrm{mspr} \mu \nu$, $\mathfrak{F}$ Rct $\mathfrak{M N}, S=\sigma \mathfrak{F}$, and

$$
\sup \mathfrak{g} \in \operatorname{cbl} \cap \operatorname{sb} \mathfrak{F}(\sigma \mathfrak{G})=b<\infty
$$

then

$$
\iint \operatorname{Cr}(x, y) \operatorname{S\nu } d y \mu d x=b
$$

Proof. So choose

$$
\text { (S) } \in \operatorname{cbl} \cap \mathrm{sb} \mathfrak{F}
$$

that

$$
\phi(\sigma(s)=b,
$$

let 


$$
T=\sigma(S,
$$

and divide the remainder of the proof into 5 parts.

PART I. $b=\int \nu($ vs $T x) \mu d x$.

Proof. Use 5.11.

PART II. If $0<r<\infty$,

$$
\begin{aligned}
A & =\mathrm{Ex}[\nu(\operatorname{vs} T x)<r<\nu(\operatorname{vs} S x)], \\
A^{\prime} & =\mathrm{Ex}[r<\nu(\operatorname{vs} S x)]
\end{aligned}
$$

then

$$
A^{\prime} \in \mathfrak{M} \cap \text { null } \mathfrak{M} \mu A .
$$

Proof. Let $t \in A^{\prime}$. Since $r<\nu($ vs $S t)$, vs $S t \in \mathfrak{N}, \nu \in C k \mathfrak{R}$ we can and do choose such a member $C^{\prime \prime}$ of Closed $\mathfrak{N}$ that

$$
C^{\prime \prime} \subset \text { vs } S t, \quad r<\nu\left(C^{\prime \prime}\right)<\infty .
$$

Let

$$
\mathfrak{F}_{1}=\mathrm{E} \beta \in \mathfrak{F}(t \in \mathrm{dmn} \beta),
$$

check that

$$
C^{\prime \prime} \subset \operatorname{rng} \sigma \mathfrak{F}_{1},
$$

and use the fact that $\nu \in$ Lind $\mathfrak{R}$ to secure such a countable subfamily $\mathfrak{S}_{1}$ of $\mathfrak{F}_{1}$ that

$$
\nu\left(C^{\prime \prime} \sim \operatorname{rng} \sigma \mathscr{S}_{1}\right)=0 .
$$

Now use the fact that $\mathfrak{R \subset} \mathrm{mbl} \nu$ and the now evident fact that

$$
\nu\left(\text { rng } \sigma \mathfrak{H}_{1}\right) \geqq \nu\left(C^{\prime \prime}\right)>r
$$

to secure such a finite subfamily $\mathfrak{S}_{1}$ of $\mathfrak{S}_{1}$ that

$$
r<\nu\left(\text { rng } \sigma \mathfrak{S}_{1}\right) \text {. }
$$

Let

$$
\begin{aligned}
& \gamma=\operatorname{rct}\left(\cap \beta \in \mathfrak{W}_{1} \operatorname{dmn} \beta\right)\left(\bigcup \beta \in \mathfrak{W}_{1} \operatorname{rng} \beta\right), \\
& \alpha=\operatorname{dmn} \gamma .
\end{aligned}
$$

Note that

$$
t \in \alpha \in \mathfrak{M}
$$

and complete the proof in Step 4 below by verifying that

$$
\mu(\alpha A)=0 \text { and } \alpha \subset A^{\prime} .
$$

STEP 1. $\gamma \subset \sigma \mathfrak{S}_{1}$.

Proof. 


$$
\begin{aligned}
\gamma & =\bigcup \beta \in \mathfrak{W}_{1} \operatorname{rct}\left(\cap \beta \in \mathfrak{W}_{1} \operatorname{dmn} \beta\right) \operatorname{rng} \beta \\
& \subset \cup \beta \in \mathfrak{W}_{1} \operatorname{rct} \operatorname{dmn} \beta \operatorname{rng} \beta \\
& =\bigcup \beta \in \mathfrak{W}_{1} \beta=\sigma \mathfrak{W}_{1} .
\end{aligned}
$$

STEP 2. $\nu(\operatorname{rng} \gamma)>r$.

Proof. Because of (1) and the (2) fact that $\operatorname{rng} \gamma=\operatorname{rng} \sigma \mathfrak{W}_{1}$

$$
\nu(\operatorname{rng} \gamma)=\nu\left(\operatorname{rng} \sigma \mathfrak{S}_{1}\right)>r .
$$

STEP 3. $\phi(\gamma \sim T)=0$.

Proof. Let $\mathfrak{S}^{\prime}=\left(\mathfrak{S} \cup \mathfrak{S}_{1}\right.$. Thus

$$
\text { (अ) } \in \mathrm{cbl} \cap \mathrm{sb} \mathfrak{F},
$$

and, in the light of Step 1,

$$
\begin{aligned}
b & =\sup \mathfrak{W} \in \operatorname{cbl} \cap \operatorname{sb} \mathfrak{F} \phi(\sigma \mathfrak{W}) \\
& \geqq \phi\left(\sigma \mathfrak{S}^{\prime}\right) \\
& =\phi(T)+\phi\left(\sigma \mathfrak{S}^{\prime} \sim T\right) \\
& =\phi(T)+\phi\left(\sigma \mathfrak{S}_{1} \sim T\right) \\
& \geqq \phi(T)+\phi(\gamma \sim T) \\
& =b+\phi(\gamma \sim T), \\
0 & \leqq \phi(\gamma \sim T) \leqq b-b=0 .
\end{aligned}
$$

STEP 4. $\mu(\alpha A)=0$ and $\alpha \subset A^{\prime}$.

Proof. For each $x \in \alpha$ we infer from Steps 1 and 2 that

$$
r<\nu(\operatorname{rng} \gamma)=\nu(\operatorname{vs} \gamma x) \leqq \nu(\text { vs } S x)
$$

and hence that $x \in A^{\prime}$. Accordingly $\alpha \subset A^{\prime}$.

Since by Step 3

it follows that

$$
\int \nu(\operatorname{vs}(\gamma \sim T) x) \mu d x=\phi(\gamma \sim T)=0
$$

$$
\mu(\mathrm{E} x \in \alpha[\nu(\operatorname{vs}(\gamma \sim T) x)>0])=0 .
$$

But, because of Step 2,

$$
\begin{aligned}
\alpha A & =\mathrm{E} x \in \alpha[\nu(\operatorname{vs} T x)<r] \\
& \subset \mathrm{E} x \in \alpha[\nu(\operatorname{vs} T x)<\nu(\operatorname{rng} \gamma)] \\
& =\mathrm{E} x \in \alpha[\nu(\operatorname{vs} T x)<\nu(\operatorname{vs} \gamma x)] \\
& \subset \mathrm{E} x \in \alpha[\nu(\operatorname{vs}(\gamma \sim T) x)>0] .
\end{aligned}
$$

Consequently 


$$
\mu(\alpha A)=0 .
$$

PART III. If $0<r<\infty$,

$$
A=\mathrm{E} x[\nu(\operatorname{vs} T x)<r<\nu(\text { vs } S x)]
$$

then

$$
\mu(A)=0 .
$$

Proof. Let

$$
A^{\prime}=\mathrm{E} x[r<\nu(\operatorname{vs} S x)] .
$$

We know from Part II that

$$
A \subset A^{\prime} \in \mathfrak{M} \cap \text { null } \mathfrak{M} \mu A .
$$

Now if

$$
C \in \text { Closed } \mathfrak{M} \cap \mathrm{dmn}^{\prime} \mu \cap \mathrm{sb} A^{\prime}
$$

then: because of 6.10 and 6.11

$$
\mu(A C)=0 ;
$$

hence

$$
\operatorname{Alm} \mu x\{\nu(\operatorname{vs} T x) \geqq r \cdot \operatorname{Cr} x C\} ;
$$

because of this and Part I

$$
\begin{aligned}
\infty & >b=\int \nu(\operatorname{vs} T x) \mu d x \\
& \geqq r \cdot \int \operatorname{Cr} x C \mu d x=r \cdot \mu(C) ; \\
\mu(C) & \leqq b / r<\infty .
\end{aligned}
$$

Consequently, since $\mu \in \mathrm{Ck} \mathfrak{M}$,

$$
\mu\left(A^{\prime}\right) \leqq b / r<\infty
$$

and 6.12 assures us

$$
\mu(A)=\mu\left(A A^{\prime}\right)=0 .
$$

Part IV. Alm $\mu x\{\nu(\operatorname{vs} T x)=\nu($ vs $S x)\}$.

Proof. Let $R^{\prime}$ be the positive rational numbers. Clearly

$$
\begin{aligned}
\operatorname{rlm} \mu \cap \operatorname{Ex}[\nu(\operatorname{vs} T x) & \neq \nu(\operatorname{vs} S x)] \\
& =U r \in R^{\prime} \operatorname{Ex}[\nu(\operatorname{vs} T x)<r<\nu(\operatorname{vs} S x)]
\end{aligned}
$$

and the desired conclusion now follows from Part III. 
Part V. $\iint \mathrm{Cr}(x, y) S \nu d y \mu d x=b$.

Proof. From Part IV, Part I, and the fact that for each $x$

$$
\text { vs } S x \in \mathfrak{R} \subset \mathrm{mbl} \nu
$$

we infer

$$
\begin{aligned}
\infty>b & =\int \nu(\operatorname{vs} S x) \mu d x \\
& =\iint \operatorname{Cr}(x, y) S \nu d y \mu d x .
\end{aligned}
$$

7.5 Lemma. If $\mu \in \mathrm{Ck} \mathfrak{M}$ Clin $\mathfrak{M}, \nu \in \mathrm{Ck} \mathfrak{R}$ Clin $\mathfrak{R}, \phi=\operatorname{mspr} \mu \nu, \mathfrak{T}=\mathfrak{M} \square \mathfrak{N}$, $\mathfrak{F} \subset$ Ret $\mathfrak{M N}, S=\sigma \mathfrak{F}$, and

$$
\sup \beta \in \text { Closed } \mathfrak{T} \cap \mathrm{dmn}^{\prime} \phi \cap \operatorname{sb} S \phi(\beta)=a<\infty
$$

then:

$.1 \phi(S)=a$;

$.2 S \in \mathrm{mbl}^{\prime} \phi$;

.3 there is such a countable subfamily \& of $\mathfrak{F}$ that

$$
\phi(S \sim \sigma())=0 .
$$

Proof. Let

$$
b=\sup \mathfrak{S} \in \operatorname{cbl} \cap \operatorname{sb} \mathfrak{F} \phi(\sigma \mathfrak{W}),
$$

so choose

$$
\text { (S) } \in \mathrm{cbl} \mathrm{sb} \mathfrak{F}
$$

that

$$
b=\phi(\sigma()) .
$$

We divide the remainder of the proof into 6 steps. The first of these is easily checked with the aid of 5.13 , and the second is an immediate consequence of 7.4. In connection with Step 3 the reader may prefer to convince himself that an alternate proof can be given, without the use of 5.16 and 5.17, by making the obvious changes in the proof of 7.4. Conclusions $.3, .2, .1$ follow respectively from Steps 4,5 , and 6 .

STEP 1. $\phi\left(\sigma(\mathcal{S})=b \leqq a<\infty\right.$ and $\sigma\left(B \in \mathrm{mbl}^{\prime} \phi\right.$.

STEP 2. $\iint \mathrm{Cr}(x, y) \operatorname{S\nu dy} \mu d x=b$.

STEP 3. $\iint \mathrm{Cr}(x, y) S \mu d x \nu d y=b$.

Proof. Let

$$
\begin{gathered}
\mu^{\prime}=\nu, \quad \nu^{\prime}=\mu, \quad \mathfrak{M}^{\prime}=\mathfrak{N}, \quad \mathfrak{N}^{\prime}=\mathfrak{M}, \\
\phi^{\prime}=\operatorname{mspr} \nu \mu,
\end{gathered}
$$




$$
\begin{aligned}
& \mathfrak{F}^{\prime}=\bigcup \beta \in \mathfrak{F} \operatorname{sng} \operatorname{inv} \beta, \\
& S^{\prime}=\sigma \mathfrak{F}^{\prime} .
\end{aligned}
$$

Clearly,

$$
\mathfrak{F}^{\prime} \subset \operatorname{Rct} \mathfrak{M}^{\prime} \mathfrak{N}^{\prime}
$$

Furthermore, if $\Re \subset \mathfrak{F}$ then

$$
\begin{aligned}
\sigma(\bigcup \beta \in \Re \operatorname{sng} \operatorname{inv} \beta) & =\bigcup \beta \in \Re \sigma \text { sng inv } \beta \\
& =\bigcup \beta \in \Re \operatorname{inv} \beta=\operatorname{inv} \cup \beta \in \Re \beta=\operatorname{inv} \sigma \Re .
\end{aligned}
$$

Accordingly

$.4 \sigma(\cup \beta \in \Omega$ sng inv $\beta)=\operatorname{inv} \sigma \Omega$ whenever $\Omega \subset \mathfrak{F}$ and in particular

$.5 S^{\prime}=\sigma \mathfrak{F}^{\prime}=\operatorname{inv} \sigma \mathfrak{F}=\operatorname{inv} S$.

Thus because of .4 and 5.17

$\sup \mathfrak{S}^{\prime} \in \operatorname{cbl} \cap \operatorname{sb} \mathfrak{F}^{\prime} \phi^{\prime}\left(\sigma \mathfrak{S}^{\prime}\right)=\sup \mathfrak{S} \in \operatorname{cbl} \cap \operatorname{sb} \mathfrak{F} \phi^{\prime}(\sigma \cup \beta \in \mathfrak{S} \operatorname{sng}$ inv $\beta)$

$$
\begin{aligned}
& =\sup \mathfrak{S} \in \operatorname{cbl} \cap \operatorname{sb} \mathfrak{F} \phi^{\prime}(\operatorname{inv} \sigma \mathfrak{S}) \\
& =\sup \mathfrak{S} \in \operatorname{cbl} \cap \operatorname{sb} \mathfrak{F} \phi(\sigma \mathfrak{S})=b .
\end{aligned}
$$

In view of this, .5, Step 1, and 7.4 we conclude

$$
\begin{aligned}
b & =\iint \operatorname{Cr}(x, y) S^{\prime} \nu^{\prime} d y \mu^{\prime} d x \\
& =\iint \operatorname{Cr}(y, x) S \mu d y \nu d x \\
& =\iint \operatorname{Cr}(x, y) S \mu d x \nu d y .
\end{aligned}
$$

STEP 4. $\phi(S \sim \sigma())=0$.

Proof. Let $T=\sigma(S$, and $R=S \sim T$.

From Steps 2 and 3 we know

$$
\iint \operatorname{Cr}(x, y) S \mu d x \nu d y=b=\iint \operatorname{Cr}(x, y) S \nu d y \mu d x .
$$

On the other hand from Step 1 and 5.11 we learn

$$
.7 \quad \iint \operatorname{Cr}(x, y) T \mu d x \nu d y=b=\iint \operatorname{Cr}(x, y) T \nu d y \mu d x .
$$

Now since, for each $z$,

$$
\operatorname{Cr} z R=\operatorname{Cr} z S-\operatorname{Cr} z T
$$


we infer from $.6, .7$ and Step 1 that

$$
\iint \operatorname{Cr}(x, y) R \mu d x \nu d y=0=\iint \operatorname{Cr}(x, y) R \nu d y \mu d x .
$$

Thus $R \in$ nil $\mu \nu$, and because of $5.11 \phi(R)=0$.

STEP 5. $S \in \mathrm{mbl}^{\prime} \phi$ and $\phi(S)=b$.

Proof. Use Steps 1 and 4.

Step 6. $\phi(S)=a$.

Proof. With the help of Steps 1 and 5 conclude

$$
\phi(S)=b \leqq a \leqq \phi(S) .
$$

7.6 Lemma. If $\mu \in \operatorname{Clin} \mathfrak{M}, \nu \in \mathrm{Clin} \mathfrak{\Re}$,

$$
\mu(\sigma \mathfrak{M})+\nu(\sigma \mathfrak{R})<\infty,
$$

then

$$
\operatorname{mspr} \mu \nu \in \operatorname{Clin}(\mathfrak{M} \square \mathfrak{R}) \text {. }
$$

Proof. Since $\mu(\sigma \mathfrak{M})<\infty$ and $\nu(\sigma \mathfrak{N})<\infty$ it follows that $\mu \in \mathrm{Ck} \mathfrak{M}$ Clin $\mathfrak{M}$, $\nu \in \mathrm{Ck} \mathfrak{N}$ Clin $\mathfrak{N}$. Let $\mathfrak{T}=\mathfrak{M} \square \mathfrak{N}, \phi=$ mspr $\mu \nu$ and complete the proof in five parts.

PART I. $\mathfrak{I} \subset \mathrm{mbl} \phi$.

Proof. Apply 7.5.2.

PART II. If $S \in \mathfrak{T}$ then $\phi(S)=\sup \beta \in$ Closed $\mathfrak{T} \cap \operatorname{sb} S \phi(\beta)$.

Proof. Apply 7.5.1.

PART III. $\phi \in$ Core $\mathfrak{T}$.

Proof. Let $\mathfrak{F}^{\prime}=$ Closed $\mathfrak{T}$ and, from Part I and Part II and the fact that $\phi(\sigma \mathfrak{T})<\infty$, infer $\mathfrak{F}^{\prime} \subset \mathrm{mbl} \phi$ and $\mathrm{cmpl} \mathfrak{F}^{\prime}=\mathfrak{T} \subset \operatorname{prxn} \phi \mathfrak{F}^{\prime}$. Now apply 3.5.4 to learn that

$$
\phi \in \text { Mass } \mathfrak{F}^{\prime}=\text { Core } \mathfrak{T} .
$$

PART IV. $\phi \in$ Lind $\mathfrak{T}$.

Proof. Apply 7.5.3 and 6.5.1.

PART V. $\phi \in$ Clin $\mathfrak{T}$.

Proof. Apply Parts III and IV.

7.7 Theorem. If $\mu \in \mathrm{Clin} \mathfrak{M}, \nu \in \mathrm{Clin} \mathfrak{\Re}$, then

$$
\operatorname{mspr} \mu \nu \in \operatorname{Clin}(\mathfrak{M} \square \mathfrak{N}) \text {. }
$$

Proof. Let $\mathfrak{T}=\mathfrak{M} \square \mathfrak{N}, \phi=$ mspr $\mu \nu, \mathcal{S}=\sigma \mathfrak{T}$.

The desired conclusion may be established by noticing that if we view Parts I, II, III below, in the light of assumption (1), then:

$$
\mathfrak{I} \subset \mathrm{mbl} \phi \text {; }
$$

for each $D \in \mathfrak{T}$ and each $T \in \mathrm{dmn}^{\prime} \phi$, 


$$
\inf C \in \text { Closed } \mathfrak{T} \cap \operatorname{sb} D \text { sct } \phi T(D \sim C)=0 \text {; }
$$

$$
\phi \in \text { Lind } \mathfrak{T} \text {. }
$$

Assume

$$
\phi(T)<\infty, \quad r>0, \quad D \in \mathfrak{T}, \quad \mathfrak{F} \subset \mathfrak{T}, \quad \mathcal{S}=\sigma \mathfrak{F} .
$$

Use 5.14.2 to secure such a member $T^{\prime}$ of bscrct $\mu \nu$ that

$$
\phi\left(T \sim T^{\prime}\right) \leqq r / 2 .
$$

Let $\psi=\operatorname{sct} \phi T^{\prime}$ and so choose $A \in \mathrm{mbl}^{\prime} \mu$ and $B \in \mathrm{mbl}^{\prime} \nu$ that $T^{\prime}=\operatorname{rct} A B$. By 5.15

$$
\psi=\operatorname{mspr}(\operatorname{sct} \mu A)(\operatorname{sct} \nu B) .
$$

Also

$$
\begin{aligned}
& \text { sct } \mu A \in \text { Clin } \mathfrak{M}, \quad \text { sct } \nu B \in \text { Clin } \mathfrak{R}, \\
& \text { sct } \mu A(\sigma \mathfrak{M})+\text { sct } \nu B(\sigma \mathfrak{R})<\infty,
\end{aligned}
$$

and hence by 7.6 and (3)

(4)

$$
\psi \in \text { Clin } \mathfrak{T} .
$$

PART I. $\phi(T)+r \geqq \phi(T D)+\phi(T \sim D)$.

Proof. Using (2) and (4),

$$
\begin{aligned}
\phi(T D)+\phi(T \sim D) & \leqq \phi\left(T^{\prime} T D\right)+\phi\left(T^{\prime} T \sim D\right)+\phi\left(T \sim T^{\prime}\right)+\phi\left(T \sim T^{\prime}\right) \\
& \leqq \phi\left(T^{\prime} T D\right)+\phi\left(T^{\prime} T \sim D\right)+r \\
& =\psi(T D)+\psi(T \sim D)+r \\
& =\psi(T)+r \\
& =\phi\left(T^{\prime} T\right)+r \\
& \leqq \phi(T)+r .
\end{aligned}
$$

PART II. There exists $C \in$ Closed $\mathfrak{T}$ such that

$$
C \subset D \text { and } \operatorname{set} \phi T(D \sim C) \leqq r .
$$

Proof. Use (4) to secure $C \in$ Closed $\mathfrak{T}$ for which $C \subset D$ and $\psi(D \sim C) \leqq r / 2$. Thus by (2),

$$
\begin{aligned}
\operatorname{sct} \phi T(D \sim C) & =\phi(T D \sim C) \\
& \leqq \phi\left(T^{\prime} D \sim C\right)+\phi\left(T \sim T^{\prime}\right) \\
& =\psi(D \sim C)+\phi\left(T \sim T^{\prime}\right) \\
& \leqq r / 2+r / 2 \\
& =r .
\end{aligned}
$$

PART III. There exists such a countable subfamily \& of $\mathfrak{F}$ that 


$$
\text { sct } \phi T(S \sim \sigma(S) \leqq r .
$$

Proof. Use (4) to secure such a countable subfamily $\mathfrak{B}$ of $\mathfrak{F}$ that

$$
\psi(S \sim \sigma(S)=0 .
$$

Thus

$$
\begin{aligned}
\operatorname{sct} \phi T(S \sim \sigma(S) & =\phi(T \sim \sigma(S) \\
& \leqq \phi\left(T^{\prime} \sim \sigma(S)+\phi\left(T \sim T^{\prime}\right)\right. \\
& =\psi\left(S \sim \sigma(S)+\phi\left(T \sim T^{\prime}\right)\right. \\
& \leqq 0+r / 2 \\
& \leqq r .
\end{aligned}
$$

To point out the utility of 7.7 we should like to formulate in 7.9 two immediate special consequences of it and 5.11.

7.8 Definition. Borelian $\mathfrak{T}=\mathrm{E} f[\mathfrak{T} \in$ topology, $f$ is such a function on $\sigma \mathfrak{T}$ to the reals that

$$
\operatorname{Ex}[f(x) \geqq \lambda] \in \text { Borel } \mathfrak{I}
$$

whenever $-\infty \leqq \lambda \leqq \infty]$.

7.9 ThEOREMS.

.1 If $\mu \in$ Clin $\mathfrak{M}, \nu \in C$ lin $\mathfrak{R}$,

$$
\begin{aligned}
& \mu(\sigma \mathfrak{M})+\nu(\sigma \mathfrak{R})<\infty, \\
& f \in \text { Borelian }(\mathfrak{M} \square \mathfrak{N}), \\
& f(z) \geqq 0
\end{aligned}
$$

then

$$
\iint f(x, y) \mu d x \nu d y=\iint f(x, y) \nu d y \mu d x .
$$

.2 If $\mu \in$ Clin $\mathfrak{M}, \nu \in C$ lin $\mathfrak{R}, \phi=\operatorname{mspr} \mu \nu$,

$$
\begin{aligned}
& f \in \text { Borelian }(\mathfrak{M} \square \mathfrak{N}), \\
& f(z) \geqq 0 \\
& \mathrm{Ez}[f(z)>0] \in \mathrm{cmbl} \phi,
\end{aligned}
$$

then

$$
\iint f(x, y) \mu d x \nu d y=\iint f(x, y) \nu d y \mu d x \text {. }
$$

7.10 Theorem. If $\mu \in \mathrm{Ck} \mathfrak{M}$ Clin $\mathfrak{M}, \nu \in C k \mathfrak{N}$ Clin $\mathfrak{N}, \mathfrak{T}=\mathfrak{M} \square \mathfrak{N}$ then 


$$
\operatorname{mspr} \mu \nu \in \mathrm{Ck} \mathfrak{T} \operatorname{Clin} \mathfrak{T} \text {. }
$$

Proof. It follows from 7.7 that

$$
\operatorname{mspr} \mu \nu \in \operatorname{Clin} \mathfrak{T}
$$

and it follows from 7.5.1 that

$$
\operatorname{mspr} \mu \nu \in \mathrm{Ck} \mathfrak{T} \text {. }
$$

7.11 Lemma. If $\mu \in \mathrm{Bh} \mathfrak{M}$ Core $\mathfrak{M}, \nu \in B$ h $\mathfrak{R}$ Core $\mathfrak{N}, \mathfrak{T}=\mathfrak{M} \square \mathfrak{N}$, $\psi \in \operatorname{Mspr} \mu \nu \mathfrak{F}, \mathfrak{T} \subset \operatorname{mbl} \psi, S \in$ bscrct $\mu \nu, S \subset S^{\prime \prime} \in \mathfrak{F}$, then

$$
S \in \mathrm{mbl}^{\prime} \psi \text {. }
$$

Proof. First choose $a \in \mathrm{mbl}^{\prime} \mu$ and $b \in \mathrm{mbl}^{\prime} \nu$ so that

$$
S=\operatorname{rct} a b
$$

and then either directly or with the help of 6.3 .3 secure $a^{\prime}, a^{\prime \prime}, b^{\prime}, b^{\prime \prime}$, so that

$$
\begin{gathered}
a^{\prime} \in \text { Borel } \mathfrak{M}, \quad a^{\prime \prime} \in \text { Borel } \mathfrak{M}, \quad a^{\prime} \subset a \subset a^{\prime \prime}, \\
\mu\left(a^{\prime}\right)=\mu(a)=\mu\left(a^{\prime \prime}\right), \\
b^{\prime} \in \text { Borel } \mathfrak{N}, \quad b^{\prime \prime} \in \text { Borel } \mathfrak{N}, \quad b^{\prime} \subset b \subset b^{\prime \prime}, \\
\nu\left(b^{\prime}\right)=\nu(b)=\nu\left(b^{\prime \prime}\right) .
\end{gathered}
$$

Now

$$
\begin{aligned}
& \operatorname{rct} a^{\prime} b^{\prime} \in \text { Borel } \mathfrak{T} \subset \operatorname{mbl} \psi, \\
& \operatorname{rct} a^{\prime \prime} b^{\prime \prime} \in \text { Borel } \mathfrak{T} \subset \operatorname{mbl} \psi, \\
& \operatorname{rct} a^{\prime} b^{\prime} \subset S \subset S^{\prime \prime} \operatorname{rct} a^{\prime \prime} b^{\prime \prime} \subset S^{\prime \prime} \in \operatorname{cmbl} \psi .
\end{aligned}
$$

Consequently, in'view of 5.5.1 and 5.3 we have

$$
\begin{aligned}
\mu\left(a^{\prime}\right) \cdot \nu\left(b^{\prime}\right) & =\iint \operatorname{Cr}(x, y) \operatorname{rct} a^{\prime} b^{\prime} \mu d x \nu d y \\
& =\psi\left(\operatorname{rct} a^{\prime} b^{\prime}\right) \leqq \psi(S) \leqq \psi\left(S^{\prime \prime} \operatorname{rct} a^{\prime \prime} b^{\prime \prime}\right) \\
& =\iint \operatorname{Cr}(x, y)\left(S^{\prime \prime} \operatorname{rct} a^{\prime \prime} b^{\prime \prime}\right) \mu d x \nu d y \\
& \leqq \iint \operatorname{Cr}(x, y) \operatorname{rct} a^{\prime \prime} b^{\prime \prime} \mu d x \nu d y=\mu\left(a^{\prime \prime}\right) \cdot \nu\left(b^{\prime \prime}\right) \\
& =\mu\left(a^{\prime}\right) \cdot \nu\left(b^{\prime}\right)<\infty .
\end{aligned}
$$

Thus

$$
\begin{aligned}
\psi\left(S \sim \operatorname{rct} a^{\prime} b^{\prime}\right) & \leqq \psi\left(S^{\prime \prime} \operatorname{rct} a^{\prime \prime} b^{\prime \prime} \sim \operatorname{rct} a^{\prime} b^{\prime}\right) \\
& =\psi\left(S^{\prime \prime} \operatorname{rct} a^{\prime \prime} b^{\prime \prime}\right)-\psi\left(\operatorname{rct} a^{\prime} b^{\prime}\right)=0
\end{aligned}
$$


and

$$
S=\operatorname{rct} a^{\prime} b^{\prime} \cup S \sim \operatorname{rct} a^{\prime} b^{\prime} \in \operatorname{mbl}^{\prime} \psi .
$$

7.12 Theorem. If $\mu \in \mathrm{Bh} \mathfrak{M}$ Clin $\mathfrak{M}, \nu \in \mathrm{Bh} \mathfrak{N}$ Clin $\mathfrak{N}, \phi=\operatorname{mspr} \mu \nu$, $\psi=$ msprb $\mathfrak{M N} \mathfrak{N}, \mathfrak{T}=\mathfrak{M} \square \mathfrak{N}$, then

$.1 \phi(\alpha)=\psi(\alpha)$ whenever $\alpha \in$ Borel $\mathfrak{T}$,

$.2 \psi \in \mathrm{Bh} \mathfrak{T}$ Clin $\mathfrak{T}$ Fubini $\mu \nu$.

Proof. Let $\mathfrak{S}=$ Borel $\mathfrak{T}$, deduce .1 from 6.8 .8 , and use 6.8.9 and 6.8.12 to see that

$$
\psi \in \operatorname{Bh} \mathfrak{T} \operatorname{Clin} \mathfrak{T}
$$

Now let

$$
\mathfrak{F}=\text { Borel } \mathfrak{T} \mathrm{dmn}^{\prime} \phi
$$

Clearly

$$
\psi=\operatorname{mss} \phi \sigma \mathfrak{T S}=\operatorname{mss} \phi \sigma \mathfrak{T} \mathfrak{F}=\operatorname{mss} \psi \sigma \mathfrak{T} \mathfrak{F}
$$

Consequently

$$
\mathfrak{F} \in \mathrm{bsc} \psi
$$

and from .1 and 5.11 we see that

$.3 \psi \in \operatorname{Mspr} \mu \nu \mathfrak{F}$.

Since $\mathfrak{T} \subset \mathrm{mbl} \psi$ and since any $S \in$ bscrct $\mu \nu$ is clearly a subset of an $S^{\prime \prime} \in($ bscrct $\mu \nu) \cap ₹$ we learn from 7.11 that

$$
S \in \mathrm{mbl}^{\prime} \psi \text {. }
$$

Consequently

$$
\text { bscrct } \mu \nu \subset \mathrm{mbl}^{\prime} \psi
$$

and because of $.3,5.3$, and 5.0.8 we are sure

$$
\psi \in \text { Fubini } \mu \nu \text {. }
$$

7.13 Theorem. If $\mu \in \mathrm{Oh} \mathfrak{M}$ Clin $\mathfrak{M}, \nu \in \mathrm{Oh} \mathfrak{R}$ Clin $\mathfrak{N}, \phi=\operatorname{mspr} \mu \nu$, $\psi=$ mspro $\mathfrak{M N} \mu \nu, \mathfrak{T}=\mathfrak{M} \square \mathfrak{R}$, then

$.1 \phi(\alpha)=\psi(\alpha)$ whenever $\alpha \in \mathfrak{T}$,

$.2 \psi \in$ Oh $\mathfrak{T}$ Clin $\mathfrak{T}$ Fubini $\mu \nu$.

Proof. Let $\mathfrak{E}=\mathfrak{T}$, deduce .1 from 6.8 .8 , and use 6.8 .9 and 6.8 .12 to see that

$$
\psi \in \text { Oh } \mathfrak{T} \text { Clin } \mathfrak{T}
$$

Now let 


$$
\mathfrak{F}=\mathfrak{T} \mathrm{dmn}^{\prime} \phi .
$$

Clearly

$$
\psi=\operatorname{mss} \phi \sigma \mathfrak{T} \mathfrak{W}=\operatorname{mss} \phi \sigma \mathfrak{T} \mathfrak{F}=\operatorname{mss} \psi \sigma \mathfrak{T} \mathfrak{F}
$$

Consequently

$$
\mathfrak{F} \in \operatorname{bsc} \psi
$$

and from .1 and 5.11 we see that

$$
\psi \in \operatorname{Mspr} \mu \nu \mathfrak{F} .
$$

Since $\mathfrak{T} \subset \mathrm{mbl} \psi$ and since any $S \in$ bscrct $\mu \nu$ is clearly a subset of an $S^{\prime \prime} \in($ bscrct $\mu \nu) \cap \mathfrak{F}$ we learn from 7.11 that

$$
S \in \operatorname{mbl}^{\prime} \psi \text {. }
$$

Consequently

$$
\text { bscrct } \mu \nu \subset \mathrm{mbl}^{\prime} \psi
$$

and because of $.3,5.3$, and 5.0 .8 we are sure

$$
\psi \in \text { Fubini } \mu \nu \text {. }
$$

7.14 Theorem. If $\mu \in \mathrm{Oh} \mathfrak{M} \mathrm{Ck} \mathfrak{M}$ Clin $\mathfrak{M}$ and $\nu \in \mathrm{Oh} \mathfrak{R} \mathrm{Ck} \mathfrak{R}$ Clin $\mathfrak{R}$, $\psi=$ mspro $\mathfrak{M N} \mu \nu, \mathfrak{T}=\mathfrak{M} \square \mathfrak{N}$, then

$$
\psi \in \mathrm{Oh} \mathfrak{T} \mathrm{Ck} \mathfrak{T} \text { Clin } \mathfrak{T} \text { Fubini } \mu \nu .
$$

Proof. Let $\phi=\operatorname{mspr} \mu \nu$ and take advantage of 5.14 to see that

$$
\phi \in \mathrm{Oh}^{\prime} \mathfrak{T} \text {. }
$$

Thus because of 7.10

$$
\phi \in \mathrm{Oh}^{\prime} \mathfrak{T} \mathrm{Ck} \mathfrak{T} .
$$

Now let $\mathfrak{E}=\mathfrak{T}$ and apply 6.8 .13 and 7.13 to learn that

$$
\psi \in \mathrm{Oh} \mathfrak{T} \text { Ck } \mathfrak{T} \text { Clin } \mathfrak{T} \text { Fubini } \mu \nu .
$$

7.15 Theorem. If $\mu \in$ Radonmeasure $\mathfrak{M}, \nu \in$ Radonmeasure $\mathfrak{N}, \mathfrak{T}=\mathfrak{M} \square \mathfrak{R}$, $\psi=$ mspro $\mathfrak{M} \Re \mu \nu$, then

$$
\psi \in \text { Radonmeasure } \mathfrak{T} \text { Fubini } \mu \nu .
$$

7.16 REMARKs. Roughly speaking, the situation seems to be this.

If $\mu \in$ Clin $\mathfrak{M}$ and $\nu \in C$ lin $\mathfrak{N}$, as is the case in 7.7, 7.9, 7.10 and in 7.127.15 , it turns out according to 7.7 that $\mathrm{mspr} \mu \nu$, which is defined solely in terms of $\mu$ and $\nu$, nevertheless belongs to Clin (M $\square \mathfrak{N})$. Because of 5.11 we thus have in 7.7 a powerful tool for inverting the order of integration. For those to whom this is the only question of real interest the theorems after 7.7 
will be of little concern. When $\mu$ and $\nu$ are narrowed slightly in 7.10 , our mspr $\mu \nu$ follows suit. In 7.12-7.15 wherein $\mu$ and $\nu$ are somewhat differently narrowed we were forced to tame mspr $\mu \nu$ a bit, in terms of the topologies $\mathfrak{M}$ and $\mathfrak{N}$, in order to make it reflect the new properties of $\mu$ and $\nu$. That this can be done with fair ease is because of the fact that, even though mspr $\mu \nu$ might be somewhat wild on some sets, it is quite tractable on Borel (MD $\square \mathfrak{N}$ ) or for that matter on the vastly larger class Bore Closed (MP $\square \mathfrak{N}$ ).

Theorem 7.15 has been anticipated in the literature by an interesting theorem $\left(^{(}\right)$proved by $\mathrm{R}$. E. Edwards along lines suggested, in part, by some unpublished results of J. L. B. Cooper. Theorem 7.15 is, of course, a quite special case of 7.14. Even 7.14 and 7.13 are inadequate, or at best clumsy tools for dealing with measures which assign very infinite measure to every nonvacuous open set. We have in mind here measures similar to Carathéodory linear measure in the Euclidean plane. For such measures, Theorem 7.12 seems fairly satisfactory, especially if one cares for a product measure similar to the preassigned measures $\mu$ and $\nu$. If one does not care for this but wishes to maximize his freedom in interchanging the order of integration, then, as we have indicated before, we recommend that Theorem 7.7 be employed in conjunction with 5.11 .

Sandia Corporation, Albuquerque, N. M.

UNIVERSITY OF CALIFORNIA, Berkeley, Calif.

(8) R. E. Edwards, $A$ theory of Radon measures on locally compact spaces, Acta Math. vol. 89 (1953) p. 151, Theorem 11. 